\title{
ON THE ROLE OF THE ACCRETION DISK IN BLACK HOLE DISK-JET CONNECTIONS
}

\author{
J. M. Miller ${ }^{1}$, G. G. Pooley ${ }^{2}$, A. C. Fabian ${ }^{3}$, M. A. NowaK ${ }^{4}$, R. C. Reis ${ }^{1}$, E. M. CacketT ${ }^{3}$, K. Pottschmidt ${ }^{5,6}$, And J. Wilms ${ }^{7}$ \\ ${ }^{1}$ Department of Astronomy, University of Michigan, 500 Church Street, Ann Arbor, MI 48109, USA; jonmm@ @mich.edu \\ ${ }^{2}$ Cavendish Laboratory, University of Cambridge, JJ Thomson Avenue, Cambridge CB3 OHE, UK \\ ${ }^{3}$ Institute of Astronomy, University of Cambridge, Madingley Road, Cambridge CB3 0HA, UK \\ ${ }^{4}$ Kavli Institute for Astrophysics and Space Research, MIT, 77 Massachusetts Avenue, Cambridge, MA 02139, USA \\ ${ }^{5}$ CRESST and NASA Goddard Space Flight Center, Astrophysics Science Division, Code 661, Greenbelt, MD 20771, USA \\ ${ }^{6}$ Center for Space Science and Technology, University of Maryland Baltimore County, 1000 Hilltop Circle, Baltimore, MD 21250, USA \\ ${ }^{7}$ Dr. Karl-Remeis-Sternwarte and Erlangen Center for Astroparticle Physics, Sternwartestrasse 7, D-96049, Bamberg, Germany \\ Received 2011 December 22; accepted 2012 July 13; published 2012 August 29
}

\begin{abstract}
Models of jet production in black hole systems suggest that the properties of the accretion disk-such as its mass accretion rate, inner radius, and emergent magnetic field—should drive and modulate the production of relativistic jets. Stellar-mass black holes in the "low/hard" state are an excellent laboratory in which to study disk-jet connections, but few coordinated observations are made using spectrometers that can incisively probe the inner disk. We report on a series of 20 Suzaku observations of Cygnus X-1 made in the jet-producing low/hard state. Contemporaneous radio monitoring was done using the Arcminute MicroKelvin Array radio telescope. Two important and simple results are obtained: (1) the jet (as traced by radio flux) does not appear to be modulated by changes in the inner radius of the accretion disk and (2) the jet is sensitive to disk properties, including its flux, temperature, and ionization. Some more complex results may reveal aspects of a coupled disk-corona-jet system. A positive correlation between the reflected X-ray flux and radio flux may represent specific support for a plasma ejection model of the corona, wherein the base of a jet produces hard X-ray emission. Within the framework of the plasma ejection model, the spectra suggest a jet base with $v / c \simeq 0.3$ or the escape velocity for a vertical height of $z \simeq 20 \mathrm{GM} / \mathrm{c}^{2}$ above the black hole. The detailed results of X-ray disk continuum and reflection modeling also suggest a height of $z \simeq 20 \mathrm{GM} / \mathrm{c}^{2}$ for hard X-ray production above a black hole, with a spin in the range $0.6 \leqslant a \leqslant 0.99$. This height agrees with X-ray time lags recently found in Cygnus X-1. The overall picture that emerges from this study is broadly consistent with some jet-focused models for black hole spectral energy distributions in which a relativistic plasma is accelerated at $z=10-100 \mathrm{GM} / \mathrm{c}^{2}$. We discuss these results in the context of disk-jet connections across the black hole mass scale.
\end{abstract}

Key words: accretion, accretion disks - black hole physics - relativistic processes - X-rays: binaries

Online-only material: color figures

\section{INTRODUCTION}

Numerous models have been proposed to explain how accretion can power jets, and nearly all of them invoke magnetic fields at some level. The ubiquity of collimated outflows in accreting systems is suggestive of a common mechanism. Magnetocentrifugal acceleration, for instance, is one process that might work to launch jets in settings as different as disks around young stars and disks around supermassive black holes (see, e.g., Blandford \& Payne 1982). In this scenario, poloidal magnetic fields are anchored in the disk or disk atmosphere, gas can escape along the field lines, and shocks downstream in the flow can serve to further accelerate the material to produce a relativistic jet.

Evidence for magnetically driven flows has been found in the absorption spectra of young stars (e.g., Calvet et al. 1993), in cataclysmic variable stars (Mauche \& Raymond 2000), stellarmass black holes (e.g., Miller et al. 2006a, 2008; Kubota et al. 2007), and also inferred in some active galactic nuclei (AGNs; Kraemer et al. 2006). Imaging of the powerful relativistic jet in M87 on scales of just $50 \mathrm{GM} / \mathrm{c}^{2}$ shows a wide-angle outflow that is just starting to collimate (Junor et al. 1999), and may further support a magnetocentrifugal origin. Evidence for a helical magnetic field at the base of a jet has recently been inferred from monitoring observations of the "blazar" BL Lac object (Marscher et al. 2008).
Another prevalent model for powering relativistic jets from black holes invokes tapping the spin energy of the black hole via magnetic field lines (Blandford \& Znajek 1977). It is interesting to note that the required field geometry is the same as the disk driven magnetocentrifugal case: poloidal field lines must be anchored in the disk or disk atmosphere. Some estimates of the work done on hot cluster gas by powerful jets from supermassive black holes in the largest central galaxies suggest that black hole spin must be getting tapped (e.g., McNamara et al. 2009). The dichotomy between radio-loud and radio-quiet AGNs can also be explained in terms of black hole spin (Sikora et al. 2007), though the nature of the accretion flow itself may also contribute to this dichotomy.

Currently, there is no definitive evidence for or against black hole spin contributing to jet power in stellar-mass black holes (e.g., Fender et al. 2010; also see Miller et al. 2009b, 2011; Narayan \& McClintock 2012), though it is clear that different methods of constraining spin are in broad agreement and suggest high spin values in most systems (Miller et al. 2009b, 2011; McClintock et al. 2010; Duro et al. 2011). This situation is likely the result of some obvious difficulties. Theoretically, it is clear that a sort of "throttle" may act to tap the power of a spinning black hole. This throttle could be magnetic field strength or orientation, the mass accretion rate, a combination or ratio of these quantities, or some other physical quantity. Observationally, difficulties inherent in arranging coordinated 
$\mathrm{X}$-ray and radio observations, limited angular resolution for separating cores and knots in the radio band in the Southern Hemisphere, and the frequent absence of gas bearing the signatures of the work done by a jet all serve to complicate efforts to reveal the role of spin.

Regardless of the details, changes in the inner disk should produce changes in the jet. The "fundamental plane" of black hole accretion clearly demonstrates that jet properties respond to inflow properties (Merloni et al. 2003; Falcke et al. 2004; Gultekin et al. 2009; also see King et al. 2011; Jones et al. 2011). One limitation of these important relationships-and, indeed, many joint X-ray and radio studies of black holes-is that X-ray monitoring observations are seldom able to reveal properties of the disk. This is especially true for supermassive black holes because AGN disk emission peaks in the UV (which suffers from extinction), and disk reflection features can require relatively deep exposures (for a review, see Miller 2007; also see Nandra et al. 2007). In contrast, the nature of the disk in stellarmass black holes can be constrained in numerous ways, notably through its thermal emission (peaking in X-rays) and reflection signatures. However, shallow X-ray monitoring observations, monitoring with coarse spectral resolution, and monitoring confined to $E>2-3 \mathrm{keV}$ cannot sample disk emission well.

For several reasons, Cygnus $\mathrm{X}-1$ is an excellent source in which to study the role of the accretion disk itself in launching jets. First, it is known to continuously launch relativistic jets in the "low/hard" state (Pooley et al. 1999; Stirling et al. 2001; Wilms et al. 2006). In this state, prior studies clearly show that X-ray fluxes and radio fluxes from monitoring observations are coupled (Gallo et al. 2003). Radio flux modulations at the level of 1-2 mJy are observed at the orbital period of Cygnus X-1 (Pooley et al. 1999), but this amplitude is small compared to the scale of the variability sampled in this program. Second, Cygnus X-1 is relatively close, with a distance of $1.86 \mathrm{kpc}$ recently determined via radio parallax (Reid et al. 2011). This ensures high X-ray and radio flux levels-even in the low/hard state-and excellent spectra from soft X-rays up to very high energy X-rays. Last, spectra of Cygnus X-1 show two clear and independent signatures of the accretion disk in the low/hard state: cool thermal emission from the accretion disk and relativistic reflection including broad $\mathrm{Fe} \mathrm{K}$ emission lines (e.g., Frontera et al. 2001; Miller et al. 2002, 2006; Reis et al. 2010; Nowak et al. 2011; Duro et al. 2011).

We therefore made a series of 20 observations of Cygnus X-1 with Suzaku in 2009. The instruments aboard Suzaku provide moderate-resolution CCD spectra in soft X-rays, coupled with extremely sensitive and simultaneous hard X-ray coverage. Thus, Suzaku is able to detect the cool accretion disk, relativistic iron line, and broadband disk reflection spectrum in each observation. Contemporaneously, we made frequent monitoring observations of the source at $15 \mathrm{GHz}$ using the updated Ryle radio telescope, now known as the Arcminute Microkelvin Imager (AMI; Zwart et al. 2008).

Comptonization models were fitted to the HXD data obtained through this program by Torii et al. (2011). In this work, we report on efforts to model the X-ray spectra using both phenomenological models including simple disk continua and relativistic $\mathrm{Fe} \mathrm{K}$ lines, and more self-consistent physical disk reflection models. The results of these spectral fits-particularly those tied to the disk-were correlated with radio flux to understand how disks influence jet properties. It should be emphasized that the central goal of this program is to accurately characterize relative changes in disk and jet properties, not absolute properties of the system.

\section{OBSERVATIONS DATA REDUCTION}

\subsection{Radio Data}

The AMI arrays are located in Cambridge, UK. They consist of two aperture synthesis telescopes mainly devoted to CMB-related studies (Zwart et al. 2008). The observations described in this paper were made with the Large Array (the reconfigured and reequipped Ryle Telescope), consisting of eight $13 \mathrm{~m}$ antennas with a maximum baseline of about $120 \mathrm{~m}$, observing in the band $12-18 \mathrm{GHz}$. The angular resolution is typically 25 arcsec. Monitoring of small-diameter sources is undertaken in the manner described by Pooley \& Fender (1997) for the Ryle Telescope; observations are interleaved with those of a phase-reference calibrator, and after appropriate calibration the data for individual baselines are averaged as vectors. The inphase component then provides an unbiased estimate of the flux density of the source in question.

The flux-density scale is calibrated by regular observations of 3C48, 3C147, and 3C286. The overall scale is believed to be consistent with that used for the Very Large Array (R. A. Perley 2012, private communication) allowing for the use by AMI of a single linear polarization; all the AMI measurements are of Stokes' I+Q.

Table 1 lists the start times and flux density at $15 \mathrm{GHz}$ for 124 AMI observations made across the time span of our Suzaku observations. These observations are made on a best effort basis, and the observation spacing is sometimes irregular. This small downside is outweighed by the typical high cadence of these observations and their sensitivity.

In order to compare radio flux density to the X-ray spectral properties derived in the fits described above, the start times of the AMI and Suzaku observations were examined. The AMI radio observation most closely following each $\mathrm{X}$-ray observation was identified. In this way, AMI observations were selected for direct comparison to the 20 Suzaku observations. This ordering is based on the expectation that the X-ray-emitting inflow may help to shape the radio jet, but that any influence of the radio jet on the inflow is negligible by comparison.

The mean delay between X-ray and radio observations in this program is 2.3 days. This delay represents innumerable orbital timescales at the innermost stable circular orbit around the black hole, but it is an order of magnitude less than viscous timescales through the entire accretion disk in X-ray binaries, which is expected to set the overall character of the accretion flow geometry (e.g., Esin et al. 1997).

\subsection{Suzaku XIS Data}

Table 2 lists some basic parameters of the Suzaku observations obtained through this program in 2009, including the XIS spectra utilized from each observation.

Each Suzaku/XIS camera has a single CCD in a square array of 1024 by 1024 pixels. The CCDs are 18 arcmin on each side. The XIS0 and XIS3 cameras are front-illuminated CCDs, while XIS1 is back illuminated. The XIS2 camera is no longer functional due to a micrometeoroid impact. The breadth of the telescope point-spread function (PSF) - approximately 2.0 arcmin (half-power diameter) - is helpful in spreading the flux of a bright incident source over many pixels. In addition, the XIS cameras are equipped with operational modes that can 
Table 1

Radio Observations and Flux Densities

\begin{tabular}{|c|c|c|c|}
\hline MJD & $\begin{array}{c}F_{15} \\
(\mathrm{mJy})\end{array}$ & MJD & $\begin{array}{c}F_{15} \\
(\mathrm{mJy})\end{array}$ \\
\hline 54895.4000244 & $11.2(2)$ & 55080.7900391 & $12.6(2)$ \\
\hline 54899.3200073 & $7.2(1)$ & 55081.8100586 & $17.2(2)$ \\
\hline 54901.5599976 & $14.0(2)$ & 55085.0500488 & $10.6(1)$ \\
\hline 54903.2999878 & 11.1(1) & 55086.8699951 & $15.4(1)$ \\
\hline 54906.2299805 & $8.7(2)$ & 55089.6800537 & $11.4(4)$ \\
\hline 54907.5499878 & $13.2(5)$ & 55090.8699951 & $12.0(2)$ \\
\hline 54910.2399902 & $11.4(1)$ & 55092.7700195 & $11.9(2)$ \\
\hline 54912.2500000 & $8.9(2)$ & 55095.9399414 & $8.7(1)$ \\
\hline 54913.1900024 & $13.8(2)$ & 55099.7500000 & $12.8(2)$ \\
\hline 54917.2899780 & $13.2(1)$ & 55101.7299805 & $12.1(1)$ \\
\hline 54919.3099976 & $15.0(1)$ & 55105.6999512 & $15.3(1)$ \\
\hline 54920.2299805 & $14.3(2)$ & 55106.6700439 & $10.1(2)$ \\
\hline 54923.3400269 & $14.1(2)$ & 55107.8499756 & $9.3(2)$ \\
\hline 54928.3200073 & $9.3(2)$ & 55108.8000488 & $11.2(1)$ \\
\hline 54933.1900024 & $11.6(1)$ & 55109.9300537 & $13.3(2)$ \\
\hline 54934.2899780 & $13.6(2)$ & 55113.8000488 & $8.7(2)$ \\
\hline 54940.1400146 & $8.8(1)$ & 55114.6700439 & $12.8(2)$ \\
\hline 54945.1699829 & $10.8(1)$ & 55117.6800537 & $10.2(1)$ \\
\hline 54947.1500244 & $12.3(2)$ & 55119.6800537 & $13.7(1)$ \\
\hline 54948.2199707 & $12.3(1)$ & 55121.8800049 & $16.3(2)$ \\
\hline 54953.4000244 & $10.2(2)$ & 55122.9300537 & $10.0(1)$ \\
\hline 54954.4000244 & $10.6(2)$ & 55123.9200439 & $9.1(1)$ \\
\hline 54956.4199829 & $12.5(4)$ & 55124.5300293 & $8.6(7)$ \\
\hline 54958.0599976 & $9.6(1)$ & 55125.6199951 & $9.4(8)$ \\
\hline 54960.3800049 & $11.0(2)$ & 55129.9000244 & $5.9(2)$ \\
\hline 54961.3800049 & $8.8(2)$ & 55130.9000244 & $6.5(1)$ \\
\hline 54962.4000244 & $12.2(2)$ & 55131.7900391 & $6.4(2)$ \\
\hline 54963.3900146 & $17.3(1)$ & 55132.9000244 & $9.1(1)$ \\
\hline 54971.1199951 & $13.5(1)$ & 55133.8900146 & $10.5(2)$ \\
\hline 54972.4000244 & $15.7(7)$ & 55136.8499756 & $6.8(1)$ \\
\hline 54976.2899780 & $13.1(3)$ & 55137.8800049 & $10.6(2)$ \\
\hline 54983.3200073 & $14.7(2)$ & 55138.5100098 & $10(2)$ \\
\hline 54987.1300049 & $19.6(1)$ & 55141.5100098 & $7.2(3)$ \\
\hline 54989.3099976 & $23.0(6)$ & 55143.7199707 & $9.3(1)$ \\
\hline 54994.2000122 & $15.3(3)$ & 55145.8299561 & $5.1(8)$ \\
\hline 54998.2199707 & $26.7(3)$ & 55146.8399658 & $6.1(2)$ \\
\hline 54999.2000122 & $29.3(2)$ & 55147.8699951 & $5(1)$ \\
\hline 55000.1500244 & $16.7(2)$ & 55148.4799805 & $8(2)$ \\
\hline 55001.2600098 & $17.9(2)$ & 55150.8800049 & $10.1(1)$ \\
\hline 55002.2600098 & $23.1(2)$ & 55152.6400146 & $6.0(1)$ \\
\hline 55004.2800293 & $25.8(3)$ & 55155.5600586 & $10.9(6)$ \\
\hline 55005.2500000 & $24.2(3)$ & 55158.5200195 & $11.6(3)$ \\
\hline 55008.0700073 & $23.9(4)$ & 55161.4499512 & $13.3(2)$ \\
\hline 55011.1199951 & $21.5(2)$ & 55162.4899902 & $12.8(2)$ \\
\hline 55011.9500122 & $17.9(2)$ & 55163.7299805 & $18.1(1)$ \\
\hline 55012.8800049 & $13.5(3)$ & 55166.6600342 & $13.6(1)$ \\
\hline 55014.1199951 & $22.0(2)$ & 55168.6899414 & $7(1)$ \\
\hline 55015.2299805 & $20.4(3)$ & 55176.4699707 & $16.2(2)$ \\
\hline 55016.1199951 & $15.3(1)$ & 55178.7600098 & $12.8(1)$ \\
\hline 55020.1699829 & $16.8(1)$ & 55187.7399902 & $15.3(5)$ \\
\hline 55035.1099854 & $11.5(1)$ & 54965.1300049 & $13.9(1)$ \\
\hline 55043.9699707 & $14.9(1)$ & 55003.0999756 & $26.6(1)$ \\
\hline 55046.0699463 & $12.7(2)$ & 55017.0999756 & $13.7(1)$ \\
\hline 55047.0300293 & $16.4(4)$ & 55063.7199707 & $14.3(2)$ \\
\hline 55050.0600586 & $12(1)$ & 55085.9100342 & $13.6(1)$ \\
\hline 55052.0400391 & $18.3(8)$ & 55176.5899658 & $14.5(1)$ \\
\hline 55055.9100342 & $13.6(2)$ & 55196.4599609 & $14.4(2)$ \\
\hline 55058.9100342 & $14.0(1)$ & 55194.4699707 & $14.0(5)$ \\
\hline 55063.0400391 & $13.7(2)$ & 55193.4599609 & $16.7(1)$ \\
\hline 55072.8800049 & $6.6(1)$ & 55192.6700439 & $12.5(1)$ \\
\hline 55076.6899414 & $7(1)$ & 55190.6099854 & $12.0(2)$ \\
\hline 55078.9200439 & 11.1(1) & 55189.4799805 & $16.9(3)$ \\
\hline
\end{tabular}

Notes. High-resolution imaging of Cygnus X-1 shows that it has a compact jet in its low/hard state (Stirling et al. 2001). In our 2009 campaign, the activity of this jet was traced through its $15 \mathrm{GHz}$ flux density, as measured in frequent monitoring observations made with the AMI. The start times and flux densities measured in AMI observations that spanned our Suzaku campaign are given above.
Table 2

X-ray Observations

\begin{tabular}{llcc}
\hline \hline MJD & Month, Day & XIS used & $\begin{array}{c}\text { Exposure } \\
(\mathrm{ks})\end{array}$ \\
\hline 54924.0537384 & Apr 3 & $0+3$ & 15.5 \\
54929.2562847 & Apr 8 & $0+3$ & 13.1 \\
54935.7650463 & Apr 14 & $0+3$ & 13.0 \\
54944.1674769 & Apr 23 & 1 & 16.3 \\
54949.7099884 & Apr 28 & $0+3$ & 12.4 \\
54957.7006829 & May 6 & $0+3$ & 15.3 \\
54971.0242593 & May 20 & $0+3$ & 17.5 \\
54976.3582060 & May 25 & $0+3$ & 16.1 \\
54980.4956713 & May 29 & $0+3$ & 25.7 \\
54984.4814005 & Jun 2 & $0+3$ & 15.0 \\
54986.8208333 & Jun 4 & $0+3$ & 6.8 \\
55125.3772107 & Oct 21 & $0+3$ & 15.1 \\
55130.2652315 & Oct 26 & $0+3$ & 20.0 \\
55138.8946181 & Nov 3 & $0+3$ & 14.1 \\
55145.8193982 & Nov 10 & $0+3$ & 18.2 \\
55152.2855093 & Nov 17 & $0+3$ & 18.7 \\
55159.5142245 & Nov 24 & $0+3$ & 14.0 \\
55166.2922454 & Dec 1 & $0+3$ & 19.8 \\
55173.6473495 & Dec 8 & 0 & 17.3 \\
55182.0619329 & Dec 17 & 0 & 3.4 \\
\hline & & & \\
\hline
\end{tabular}

Notes. List of the start times of our 2009 Suzaku observations of Cygnus X-1 in UT, as well as the calendar day on which the observation was made, which XIS units were used in spectral fits, and the total exposure time. The start times and exposure times are drawn from the HXD/pin event files as multiple XIS event files exist owing to frequent switching between editing modes.

further aid in the observation of bright sources. The key issue is to limit photon pile-up, which can falsely reduce the flux level that is recorded and distort the spectrum by registering multiple incident photons as single events. The key quantity is the photon flux per event box (typically $3 \times 3$ pixel boxes used to select good X-ray events and reject cosmic rays) per CCD frame time (see, e.g., Miller et al. 2010). Cygnus X-1 is a very bright source, and the instrumental set-up was chosen to limit the effects of photon pile-up on the resultant XIS spectra.

The HXD pointing position was used in all observations; in this configuration, vignetting then reduces the effective area, lowering the photon flux per event box. In the vast majority of observations, each camera was operated with a $1 / 4$ window plus $0.5 \mathrm{~s}$ burst option. This has the effect of limiting the frame time, reducing the photons registered in each event box per clock time, and again limiting pile-up. In a few cases, one of the cameras was run in "PSUM" mode, which offers very high time resolution at the expense of imaging information. PSUM mode is not robust against pile-up effects for bright sources, and data taken in this mode are not considered in this work.

All Suzaku data were reduced using version 6.9 of the HEASOFT suite (including FTOOLS and CALDB). The XIS data were reduced in accordance with the Suzaku ABC Guide; these procedures and additional conservative reduction steps are briefly described below.

XIS events can be recorded and telemetered in different editing modes; $2 \times 2$ and $3 \times 3$ are the most common, and $5 \times 5$ is used less frequently. Each unfiltered event file for each camera was reprocessed using the tool "xispi." New "cleaned" event files were then made using the "xisrepro" script, run within xselect. This is often the full extent of data processing for XIS data, but we took additional steps to produce the best possible spectra. 
1. For each event file, we next ran the "aeattcor.sl" script (Nowak 2009; Nowak et al. 2011), which corrects the data for the large spacecraft wobble endemic to Suzaku. This script generates a new attitude file, which was subsequently applied to each cleaned event list. In every case, this procedure yielded a sharper PSF.

2. We then ran the XIS pile-up estimation tool (Nowak 2009; Nowak et al. 2011), and produced images with contours corresponding to different levels of photon pileup. Extraction regions were then chosen so as to limit photon pile-up. Spectra were extracted from square boxes, 240 pixels on each side, with an inner exclusion circle with a radius of 30 pixels and centered on the source coordinates. This effectively limited photon pile-up in extracted spectra to $5 \%$ or less. Backgrounds were extracted using the same region shape, offset from the center of the PSF.

In this work, we only consider spectra obtained outside of the well-known "dipping" intervals in Cygnus X-1. As shown by Balucinska-Church et al. (2000), dipping intervals vary with phase, clustering around $\phi=0$ (where the companion is closer to Earth). This clustering suggests that the dips are partially due to obscuration by inhomogeneities or "blobs" in the focused companion wind. Some dipping is seen at every orbital phase, however (again, see Balucinska-Church et al. 2000), and thus all observations must be screened for dips.

Dipping intervals were identified and excluded using the methods described by Nowak et al. (2011). Briefly, light curves and color-color diagrams were made using the XIS data, and intervals with dip-like properties were identified. These intervals were excluded by specifying additional time selections in the standard good-time interval files. This provides a relatively simple but effective means of identifying and removing dipping intervals and the spectral distortions that they introduce.

For each XIS camera, all event files were then loaded into "xselect" jointly. Regions were defined according to the prescription above. The standard good-time filtering was applied. Spectra, backgrounds, final light curves, and final good-time files were then extracted.

Redistribution matrix files were constructed for each spectrum using the tool "xisrmfgen," and ancillary response files were made by running the "xissimarfgen" tool with an input of 200,000 photons. The XIS0 and XIS3 CCDs are both frontilluminated chips, with very similar response functions. In observations where both of these CCDs were operated in imaging modes, their spectra, backgrounds, and response functions were combined using the tool "addascaspec."

It is nominally the job of the redistribution matrix file to account for oversampling of the detector energy resolution, and to handle it appropriately. This file should be built based on a statistical characterization of how often a photon-with an energy known a priori-ends up in one of $N$ channels that sample the relevant energy window. In practice, this characterization is very difficult; the mutual dependence of flux in different channels is hard to characterize perfectly. As a result, binning can tend to add more continuum into weak and/or narrow line features than is nominally correct, and thereby reduce the significance of such features. Our goal is to accurately characterize reflection in the $\mathrm{Fe} \mathrm{K}$ band, which requires separating its features from (weak and narrow) ionized absorption features. A stronger binning might legitimately reduce the statistical uncertainty in some continuum and reflection parameters, for instance, but might illegitimately reduce the complicating effects of ionized absorption in the companion wind. Although an even stronger binning would better reflect the intrinsic energy resolution of the XIS cameras, via the FTOOl "grppha" the XIS spectra were grouped to have at least 10 counts per bin for the reasons cited here.

Owing to its different response function, and its different (and generally more severe) calibration uncertainties, XIS1 was excluded from our analysis. The single exception is the observation on April 23, in which both the XIS0 and XIS3 cameras were operated in "PSUM" mode. The use of XIS1 in this case incurs a small but unavoidable systematic uncertainty relative to the other observations.

The XIS cameras suffer from calibration uncertainties at the edges of their spectral bandpass and in the $\mathrm{Si}$ region (see, e.g., Nowak et al. 2011). In order to avoid these problems, the XIS spectra were fitted in the $0.8-9.0 \mathrm{keV}$ band. In the front-illuminated XIS cameras, the largest calibration issue in the Si band can be modeled with the addition of a Gaussian absorption line at $1.86 \mathrm{keV}$. (A negative Gaussian centered at $2.44 \mathrm{keV}$ is required to account for calibration uncertainties in the XIS1 response function.) In a small number of cases, residuals in the $\mathrm{Si}$ band were markedly worse than in other observations, with instrumental flux residuals extending up to $3.5 \mathrm{keV}$. Nowak et al. (2011) noted similar problems; following that work, the $1.5-3.5 \mathrm{keV}$ band was ignored when fitting full relativistic reflection models on May 25 and 29 and June 2 and 4. These observations do not occur at the same binary phase nor do they occur at particularly high or low flux levels. This suggests a short-term change in the instrument response rather than a change in the nature of the spectra observed from Cygnus X-1. In case this interpretation is incorrect, the $1.5-3.5 \mathrm{keV}$ range was included in fits with phenomenological models.

\subsection{Suzaku HXD/Pin Data}

The HXD/pin spectra were reduced in the standard manner recommended by the $\mathrm{ABC}$ Guide. This procedure is described here for clarity.

"Tuned" background files for each observation were downloaded from the Suzaku Web site. These files take several weeks to accumulate and are available some time after an observation is made. A common good-time file was made by merging intervals from the cleaned pin event file and the non-X-ray background event file. Time filtering of the cleaned event file was then performed within "xselect" using the merged good-time file, and a pin spectrum was extracted. The resulting spectral file was corrected for dead time using the tool "hxddtcor" and the appropriate housekeeping file.

A non-X-ray background spectral file was also extracted using "xselect," after time filtering with the merged good-time file. As recommended by the ABC Guide, the exposure time of the non$\mathrm{X}$-ray background spectral file was increased by a factor of 10 using the ftool "fparkey." To create the cosmic X-ray background spectral file, the response file appropriate for the HXD pointing position and gain and calibration epoch of each observation was selected from the HXD calibration database. This file was used to simulate a spectral file within XSPEC, exactly as per the specifications in the ABC Guide. The non-X-ray background and cosmic X-ray background files were then merged using the ftool "mathpha."

In all cases, the $\mathrm{HXD} /$ pin spectra were fitted in the $12-70 \mathrm{keV}$ band. This range was found to be relatively free of strong calibration uncertainties. 


\subsection{Suzaku HXD/GSO Data}

Source spectra and non-X-ray background spectra from the $\mathrm{HXD} / \mathrm{GSO}$ were generated and corrected for dead time using the ftool "hxdgsoxbpi." The appropriate raw GSO background file for each observation was downloaded from the public archive. The spectral response files (including the "correction arf") appropriate for each observation were also downloaded from the public archive.

In all cases, the HXD/GSO spectra were fitted in the $70-500 \mathrm{keV}$ band. This range was found to be relatively free of strong calibration uncertainties. The upper limit of $500 \mathrm{keV}$ is the highest energy at which the source is detected in all of the observations.

\subsection{Correcting For Ionized Absorption}

In order to account for the effects of X-ray absorption in the companion wind, we downloaded and analyzed the longest Chandra/HETG observation in the "low/hard" state available in the public archive. Specifically, we analyzed a $55.85 \mathrm{ks}$ exposure made starting 2003 March 4 at 15:45:02 UT (sequence 400302, ObsID 3815). To prevent photon pile-up, the ACIS-S array was read-out in "continuous clocking" mode, and a gray filter was used on the S3 chip to prevent frame dropping. (For details and additional examples of this instrumental set-up, see Miller et al. 2006b, 2008).

The data were obtained using the Chandra "TGCAT" facility, which archives calibrated up-to-date, level-2 event files, spectral files, and redistribution matrix files. All processing was performed using CIAO 4.2. Using the tool "dmtype2split," we divided the delivered "pha2" file into individual components. Ancillary response files were generated using the tool "mkgarf" and combined the first-order MEG spectra and HEG spectra. These spectra were then analyzed using XSPEC.

As reported in many prior papers, the combined MEG and HEG spectra of Cygnus X-1 contain absorption lines that originate in the companion wind. The wind is not thought to be point symmetric nor even axisymmetric. Rather, comparisons from different points in the binary phase suggest that the wind is "focused" (e.g., Sowers et al. 1998; Miller et al. 2005; Gies et al. 2008; Hanke et al. 2009). The column density in the wind is maximal when the companion star is closest to the observer $(\phi=0)$, and is reduced when the black hole primary is closer to the observer $(\phi=0.5)$.

At present, no systematic, self-consistent photoionization study of spectra obtained at different binary phase points has been performed. Indeed, treatments in the literature vary widely (though the reader is referred to Hanke et al. 2009 as a thorough treatment). To address the absorption observed in this long Chandra observation, we constructed a large grid of XSTAR models using "xstar2xspec" (version 2.2). The spectral energy distribution (SED) used to create the XSTAR grid was taken from a disk blackbody plus power-law model $(k T=0.19 \mathrm{keV}$, $\Gamma=1.8$, commensurate with values found in fits to the $S u z a k u$ data) used to describe the Chandra continuum. This grid was then included in direct fits to the MEG and HEG spectra as a table model that multiplied a simple disk blackbody plus powerlaw continuum. In these fits, the ionization parameter of the absorbing gas, its equivalent neutral hydrogen column density, and its velocity shift were all free parameters.

Based on the ephemeris given by Brocksopp et al. (1999), this long Chandra observation started at a binary phase of $\phi=0.77$. Direct fitting suggests a number density of $\log (n)=11$, an

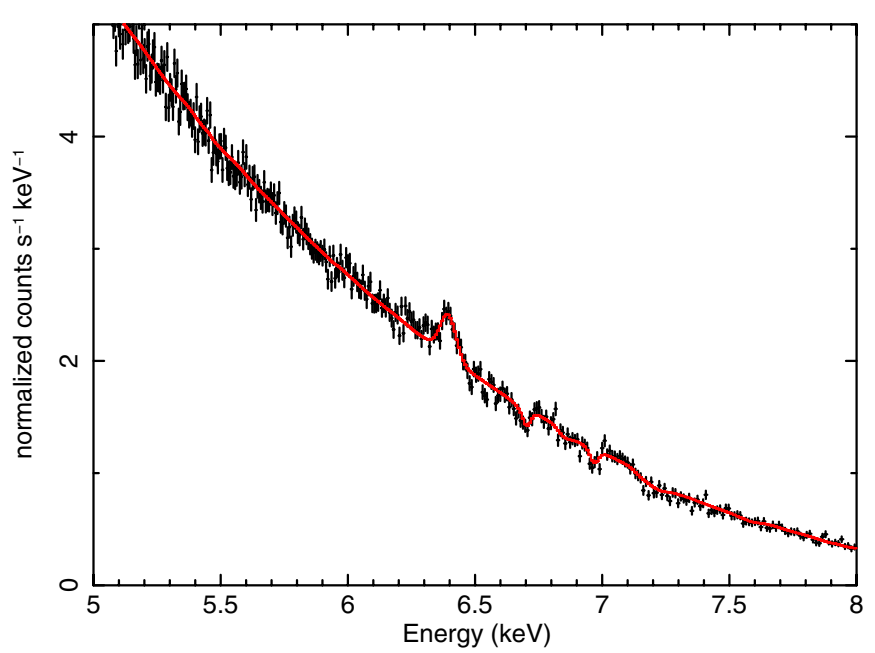

Figure 1. Deep Chandra/HETG spectrum of Cygnus X-1 in the low/hard state in 2003 . The narrow emission line at $6.40 \mathrm{keV}$ is fitted with a simple Gaussian, but the Fe XXV and Fe XXVI absorption lines at 6.70 and $6.97 \mathrm{keV}$ (respectively) are fitted with an XSTAR model with $N=4.5 \times 10^{21} \mathrm{~cm}^{-2}$ and $\log (\xi)=2.0$. This ionization parameter permits a good fit in the Fe $\mathrm{K}$ band of this observation and is commensurate with that required by many ions in a comprehensive study of wind absorption with orbital phase (Hanke et al. 2009). The same grid of ionized absorption models was applied to all Suzaku spectra of Cygnus X-1 treated in this paper.

(A color version of this figure is available in the online journal.)

ionization parameter of $\log (\xi) \simeq 2$ (where $\left.\xi=L / n r^{2}\right)$, and an equivalent neutral hydrogen column density of $N_{\mathrm{H}} \simeq 4 \times$ $10^{21} \mathrm{~cm}^{-2}$, and a modest redshift gives a reasonable description of the data (the strongest lines from abundant elements are fitted well). This is in broad agreement with other results obtained near to this phase (Miller et al. 2005; Hanke et al. 2009). In more detailed treatments, individual ions may require slightly different parameters. However, these values are representative of the results obtained even as the column density varies with binary phase (see Hanke et al. 2009). Last, it is important to note that this model correctly accounts for Fe XXV and Fe XXVI absorption lines that fall on top of the relativistic disk line in Cygnus X-1 (see Figure 1).

In all fits to the Suzaku spectra, then, this XSTAR table model was included. Although ionized absorption lines are evident in the Fe $\mathrm{K}$ band after fitting for disk reflection, the resolution of the XIS makes it difficult to clearly detect weak lines at lower energy, at least at the level required to constrain the ionization of a global model. Therefore, an ionization parameter of $\log (\xi)=2.0$ was held fixed when fitting the XSTAR table model. Moreover, the redshift of a few $\times 100 \mathrm{~km} \mathrm{~s}^{-1}$ found in fits to the Chandra/HETG spectra is insignificant at the resolution of the XIS, so a velocity shift of zero was used in all cases. Thus, only the column density was allowed to vary.

Whether or not CCD spectra could usefully constrain the parameters of an ionized absorption zone was an open question. To this end, the column density measured via phenomenological $\mathrm{X}$-ray spectral fits (see Section 3.1) is plotted versus binary phase in Figure 2. The expected variation is evident in the data: the column density is highest close to $\phi=0$ and reduced close to $\phi=0.5$. This is a strong indication that our modeling is able to correctly account for variations in the spectrum due to the companion wind, and to differentiate those variations from true changes in the low-energy disk flux and relativistic line flux. We remind the reader that dipping intervals were excluded from the spectra in this exercise and in the analysis that follows. 


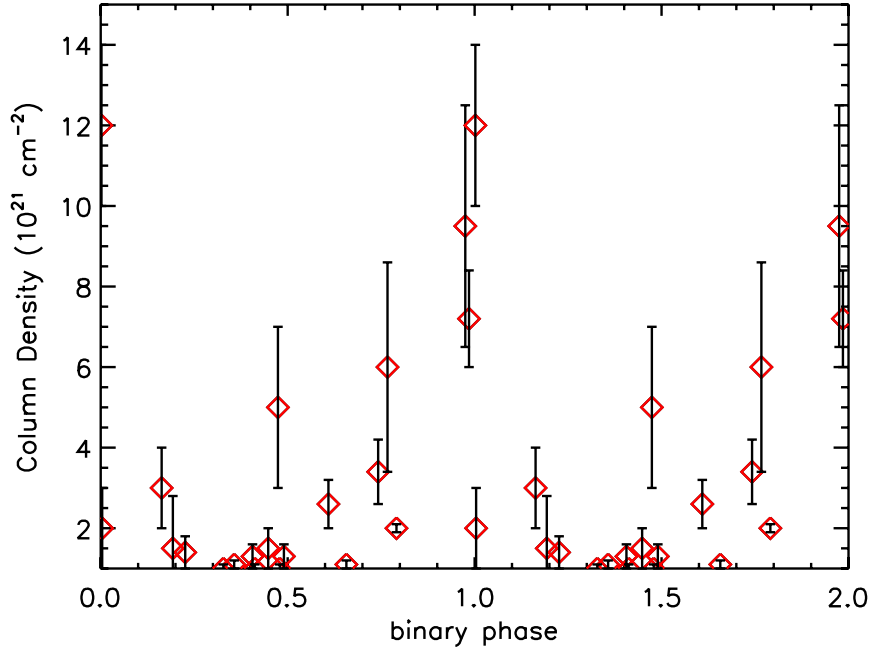

Figure 2. To account for ionized absorption in the companion wind of Cygnus $\mathrm{X}-1$, we constructed a grid of XSTAR models and included the grid as a table model with variable column density in all spectral fits. This step was important to ensure that absorption did not falsely affect the disk continua nor the Fe $\mathrm{K}$ band. The plot above shows that the ionized column varies as expected with phase: the absorption is generally strongest close to phase zero, when the $\mathrm{O}$ star companion is closest to the observer.

(A color version of this figure is available in the online journal.)

\section{X-RAY SPECTRAL MODELS}

In all fits, the equivalent neutral hydrogen column density was fixed to $N_{\mathrm{H}}=6 \times 10^{21} \mathrm{~cm}^{-2}$, as per the results of fits to high-resolution Chandra/HETG spectra (Schulz et al. 2002). Although the ionized column density in the companion wind may change as a function of orbital phase, the neutral column density is likely best modeled as being constant (Miller et al. 2009a), at least in non-dip spectra and at phases other than $\phi=0$. The "tbabs" model within XSPEC was used to fit the interstellar column density (Wilms et al. 2000), assuming the elemental abundances given by Anders \& Grevesse (1989).

As noted above, all spectral models were additionally modified by the photoionized absorber described above. The ionization parameter was fixed at $\log (\xi)=2.0$, but the equivalent hydrogen column density of this ionized absorption was allowed to vary in phenomenological fits to each observation. For simplicity and expedience, the equivalent hydrogen column density measured in each observation using phenomenological models was then held fixed when fitting the more physical reflection models.

\subsection{Phenomenological Spectral Models}

Figure 3 shows a typical broadband spectrum and the associated data/model ratio that results from fitting with a simple power-law model. The spectrum was fitted by ignoring the spectrum below $3 \mathrm{keV}$, in the Fe $\mathrm{K}$ band (taken to be $5-7 \mathrm{keV}$ here, based on typical broad emission line profiles), and in the $15-45 \mathrm{keV}$ band. The strong soft excess is evidence of thermal emission from an accretion disk, and the curvature seen through the HXD bandpass is strongly indicative of disk reflection.

Disk reflection models can differ in their assumptions. Some are especially suited to high ionization (e.g., "reflionx"; see Ross \& Fabian 2005; also see Dovciak et al. 2004), some are suited to low ionization (e.g., "pexrav"; see Magdziarz \& Zdziarski 1995). Most models assume a constant density in the upper levels of the disk where reflection occurs; this might be appropriate if, e.g., magnetic pressure dominates. Other models

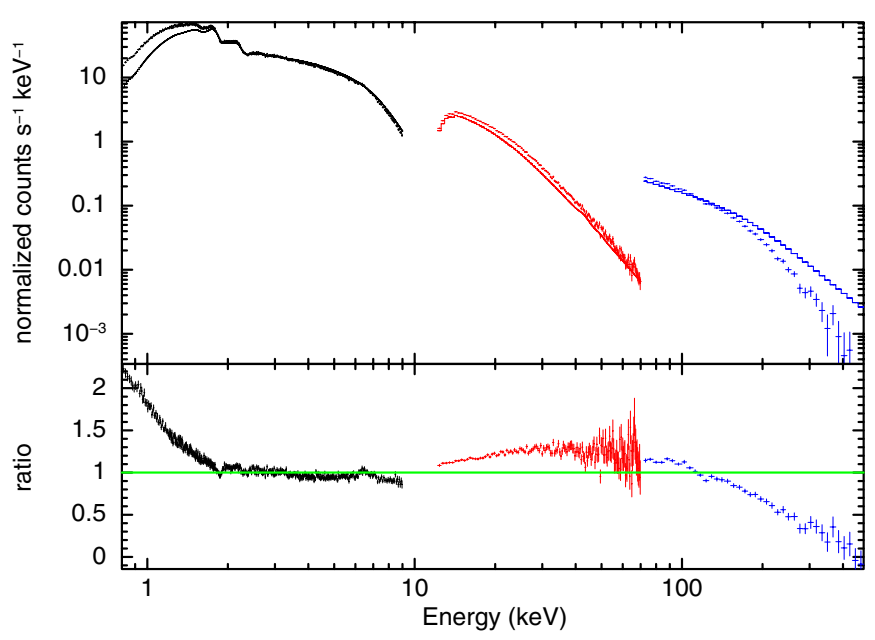

Figure 3. Panel above shows the combined backside-illuminated XIS (black) and HXD PIN (red) and GSO (blue) spectra of Cygnus X-1, obtained during an observation on 2009 May 6. The spectra have been fitted with a simple power law, ignoring the range below $3 \mathrm{keV}$, the Fe $\mathrm{K}$ band $(5-7 \mathrm{keV})$, and the $15-45 \mathrm{keV}$ band. When applied to the complete spectra extending down to $0.8 \mathrm{keV}$, a cool accretion disk continuum and disk reflection features are clearly evident. The spectra were binned for visual clarity.

(A color version of this figure is available in the online journal.)

assume a vertical density gradient determined by hydrostatic equilibrium (e.g., "xion"; see Nayakshin \& Kallman 2001).

In order to provide relatively model-independent results, then, we initially fitted every observation with a phenomenological spectral model. The model effectively replicates a relativistically blurred disk reflection spectrum with a simple continuum, but it is easily reproduced and it is free of assumptions regarding disk structure, the nature of the interaction between the corona and disk, and the physical processes at work in the corona. (The results of these fits can be compared to the Comptonization fits made to the same HXD data by Torii et al. 2011.)

The soft excess at low energy was fitted using a disk blackbody model with a modified emissivity law (the "diskpbb" model in XSPEC; e.g., Mineshige et al. 1994). This is a generalization of the better-known "diskbb" model (Mitsuda et al. 1984), in that the disk temperature is assumed to vary with radius according to $T \propto r^{-p}$ far from the black hole. The "diskbb" model assumes the "standard" value of $p=3 / 4$. In light of recent results showing that disks may be strongly irradiated by the corona in the low/hard state (Rykoff et al. 2007; Gierlinski et al. 2009; Reynolds et al. 2010; Reynolds \& Miller 2012), we have assumed $p=0.5$ as per an irradiated accretion disk. This does not bias the disk radius to be close to the black hole; rather, more of the flux is assumed to originate from larger radii.

Again, the focus of this paper is on how parameters vary, not absolute values. However, the disk continuum does permit constraints on the inner disk radii-often a parameter of interest-and we have derived radii based on the continuum as self-consistently as possible. The flux normalization of "diskbb" and "diskpbb" components is given by $K=$ $(r / \mathrm{km})^{2} \cos \theta(d / 10 \mathrm{kpc})^{-2}$. In calculating radii in gravitational units, we have assumed $d=1.86 \mathrm{kpc}$ (Reid et al. 2011), an inclination of $\theta=27^{\circ}$, and a mass of $M=14.8 M_{\odot}$ (Orosz et al. 2011). This inclination is that of the binary system; the inner disk may be misaligned (for a discussion, see Maccarone 2002), and we are simply assuming that the binary and inner disk inclinations are consistent. Given that gravitomagnetohydrodynamics tends to anchor the inner disk perpendicular to the 
black hole spin vector (Bardeen \& Petterson 1975), and that jet production should be aligned with that vector, the absence of a two-sided jet in radio images of Cygnus X-1 (Stirling et al. 2001) suggests that the inner disk is also viewed at a low inclination.

The "diskbb" model is a standard against which correction factors and calibrations have been calculated. In this analysis, the "true" inner disk radius is calculated via $\left.r_{\text {in }}=\eta f_{g r}^{2} f_{\text {col }}^{2} r_{\text {col }} / \sqrt{(} g\right)$, where $\eta$ is the ratio of the inner disk radius to the radius at which the emissivity actually peaks, $f_{g r}$ accounts for relativistic effects, and $g$ accounts for relativistic effects on apparent inclination (we have assumed $\eta=0.7, f_{g r}=0.5$, and $g=0.3$, as per Zhang et al. 1997, with the assumption that Cygnus X-1 likely has a high spin). The color correction factor, $f_{\text {col }}$, accounts for radiative transfer (we have assumed $f=1.7$ as per Shimura \& Takahara 1995 and Merloni et al. 2000). The value of $\eta$ that we have taken is likely to be marginally too high, in that it assumes a disk where $T \propto r^{-3 / 4}$ holds at large radii; a disk following $T \propto r^{-1 / 2}$ will dissipate most of its energy at even larger radii and would nominally require a smaller value of $\eta$.

The medium- and high-energy spectrum was fitted using a broken power-law component ("bknpow"), modified by an exponential cutoff at high energy ("highecut"; see Wilms et al. 2006 for background on this simple model for the high-energy spectrum of Cygnus X-1).

The Fe $\mathrm{K}$ band in Cygnus $\mathrm{X}-1$ is known to be comprised of at least two components: a narrow neutral emission line arising either though irradiation of clumps in the companion wind or the outer disk, and a broad relativistic line that is dynamically broadened in the innermost accretion disk (see, e.g., Miller et al. 2002, 2005; Nowak et al. 2011; Duro et al. 2011). The narrow, neutral line is very narrow even at HETG resolution (see Figure 1), so it was modeled using a zero-width Gaussian component with a centroid energy frozen at $6.40 \mathrm{keV}$. Although early attempts to constrain the spin parameter in Cygnus X-1 yielded inconsistent results (e.g., Miller et al. 2005, 2009b), more recent efforts using improved models and data have converged on a high spin (Gou et al. 2011; Duro et al. 2011). The broad line was therefore modeled using the "Laor" line model (Laor 1991). A drawback of the Laor model is that it assumes maximal spin; however, it allows a large range of disk radii to be explored, and the focus of this work is to understand relative rather than absolute values. The line centroid energy was bounded between 6.70 and $6.97 \mathrm{keV}$ (Fe XXV-XXVI) as per an ionized disk. The emissivity index was fixed at $q=3$ (where $J(r) \propto r^{-q}$ ); this was motivated by preliminary phenomenological and reflection fits that all returned values of $q$ consistent with 3.0. The inclination was fixed at $27^{\circ}$, again assuming that the black hole spin vector is aligned with the binary inclination (but see Maccarone 2002). The outer illumination radius was fixed at $400 \mathrm{GM} / \mathrm{c}^{2}$ and the inner radius was allowed to vary.

The total phenomenological spectral model used was

$$
\begin{gathered}
\text { const } \times \text { tbabs } \times \text { mtable }\{\text { wind_abs }\} \times(\text { gauss }+ \text { laor } \\
+ \text { diskpbb }+ \text { bknpow }) \times \text { highecut. }
\end{gathered}
$$

\subsection{Relativistically Blurred Reflection Models}

To provide a more physical description of the inner accretion flow, we also fitted each of the broadband spectra with the "reflionx" disk reflection model (Ross \& Fabian 2005; note that Comptonization models, sometimes invoking multiple Comptonization zones, may also provide a reasonable physical description of the data; see Torii et al. 2011). This model as- sumes a constant density for the accretion disk, and the emergent spectrum is averaged over viewing angle. However, it is suited to a broad range of ionization parameters; it includes emission lines from $\mathrm{C}, \mathrm{N}, \mathrm{O}, \mathrm{Ne}, \mathrm{Mg}, \mathrm{Si}, \mathrm{S}$, and $\mathrm{Fe}$; and its temperature and ionization calculations do not assume local thermodynamic equilibrium. Unlike some reflection models, "reflionx" is calculated over the $1 \mathrm{eV}$ to $1 \mathrm{MeV}$ range, so it can safely be applied across the entire $0.8-500 \mathrm{keV}$ bandpass of our Suzaku spectra. The broad range of elements and charge states considered in "reflionx" is of importance at low energy, where emission lines from reflection may blur together to create a pseudo-continuum. Fits with "reflionx," then, are crucial to resolving how much of the apparent disk emission is actually due to viscous dissipation in the disk, and how much might be due to reflection.

In fitting "reflionx," the iron abundance was fixed at the default (solar) value, and the source redshift was fixed at zero. The photon index of the power law assumed to irradiate the disk within "reflionx" was linked to the photon index of a separate, simple, unbroken power-law continuum component. The ionization parameter of the accretion disk and the flux normalization of the "reflionx" component were both allowed to float freely. The reflection spectrum was blurred using the "kdblur" model, which is based on the Laor line function. As per the phenomenological model, the inclination was fixed at $27^{\circ}$, the emissivity was fixed at $q=3$, the outer illumination radius was fixed at $400 G M / c^{2}$, and the inner disk radius was allowed to float freely. The narrow $\mathrm{Fe} \mathrm{K}$ emission line at $6.4 \mathrm{keV}$ was included as before. The soft thermal emission was again modeled using a generalized disk blackbody assuming irradiation ("diskpbb" with $p=0.5$ ), and the total spectral model was modified by a high-energy cutoff ("highecut"). The total spectral model used was

$$
\begin{aligned}
\text { const } & \times \text { tbabs } \times \text { mtable }\{\text { wind_abs }\} \times(\text { gauss }+ \text { dispkbb } \\
& + \text { pow }+ \text { kdblur } \times \text { atable }\{\text { reflion } . \text { mod }\}) \times \text { highecut } .
\end{aligned}
$$

It must be noted that the total spectral model is not entirely self-consistent. The "reflionx" model assumes an exponential cutoff with an $e$-folding energy fixed at $300 \mathrm{keV}$. This is generally a factor of two higher than is measured in fits with the phenomenological model (see below). Owing to this disparity, acceptable reflection fits cannot be obtained unless a separate cutoff is applied. This could have the effect of accounting for some curvature that is properly due to reflection instead through a spectral cutoff. The reflection results are driven by the features and sensitivity achieved below $10 \mathrm{keV}$, and then again in the 20-40 keV range (where the Compton back-scattering "hump" peaks), and less by the nature of the spectrum at and above $300 \mathrm{keV}$.

It should also be noted that "reflionx" does not measure the "reflection fraction," unlike some prior reflection models. This is partly because there is no longer a simple linear relationship between the incident and reflected flux, when ionization is included and properly treated. Rather, the albedo of the disk increases with ionization at high ionization parameters. This is even more true when the ionization balance within the disk is actually being solved by the reflection model. Moreover, the values obtained in calculating a "reflection fraction" are strongly dependent upon the energy band considered, and values obtained via blurred ionized reflection models fitted over the $0.8-500.0 \mathrm{keV}$ band would not easily map to values obtained with older, un-blurred models fit to a standard RXTE bandpass (e.g., 3-100 keV). 


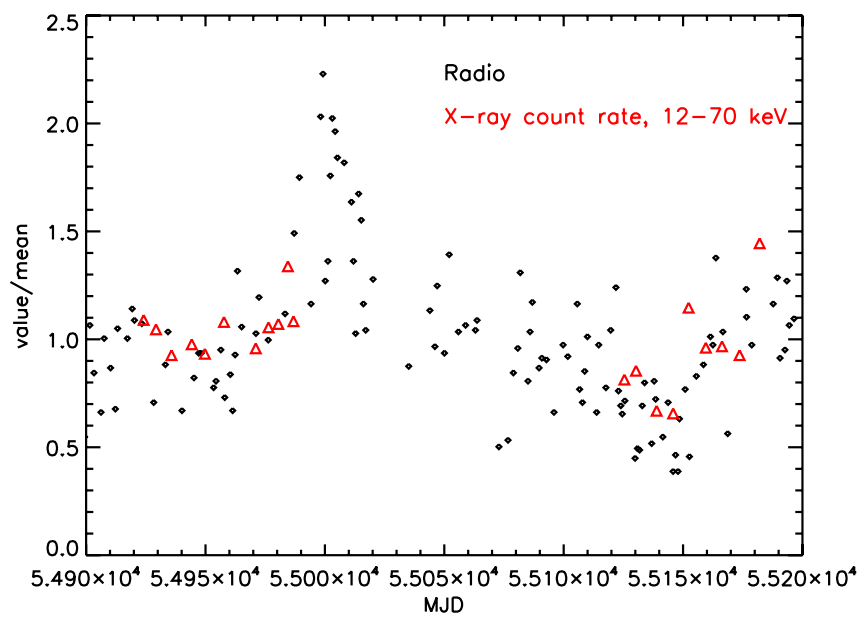

Figure 4. Time variations in the radio flux density and HXD/PIN count rate, relative to their mean values. Prior studies of disk-jet connections have essentially studied correlations between radio flux density and hard X-ray flux rather than disk properties, and these quantities are clearly correlated in our Suzaku observations.

(A color version of this figure is available in the online journal.)

Last, the "Laor" relativistic line model (Laor 1991) and the "kdblur" convolution model based on "Laor" enable relatively quick spectral fits and are sufficient to characterize relative differences in line properties. Newer line models that include spin as a variable parameter are now available (e.g., Dovciak et al. 2004; Beckwith \& Done 2004; Brenneman \& Reynolds 2006). Although such models might be better suited to measuring absolute values, they can greatly increase the time required to make a fit. New line models appear to give improvements over older models at the $10 \%$ level (Beckwith \& Done 2004).

\section{X-RAY SPECTRAL FITTING RESULTS}

\subsection{Initial Checks}

Prior to interpreting the results of X-ray spectral fits, and the implications of correlations between X-ray fitting parameters and radio flux density, the $\mathrm{X}$-ray and radio data were checked for consistency with prior results in a model-independent fashion. The $\mathrm{HXD} /$ pin count rate was selected for comparison, to best approximate the kind of data that are obtained in typical X-ray monitoring observations. Figure 4 shows light curves of the radio flux density and HXD/pin count rates; the data were divided by their mean values in order to show the relative strength of flux variations. In order to put the X-ray observations in a broader context, the full set of 124 radio observations listed in Table 1 is plotted in Figure 4. It is clear from this figure that the radio and hard X-ray points track each other well, and that the radio flux shows higher fractional variability than the X-ray flux.

Figure 5 plots radio flux density versus the $\mathrm{HXD} /$ pin count rate, for the 20 Suzaku observations and the radio observations that followed most closely after each. The quantities are clearly correlated, though there is scatter. A Spearman's rank correlation test gives a coefficient of $\rho=0.45$, corresponding to a $4.4 \%$ chance of a false correlation. Thus, the Suzaku and AMI data confirm the radio-X-ray coupling that has been seen previously with X-ray monitor data (see Gallo et al. 2003; Wilms et al. 2006; Zdziarski et al. 2011). This provides a measure of confidence that our program has sampled Cygnus $\mathrm{X}-1$ in a period of typical disk-jet coupling. The prior demonstrations of a positive radio and X-ray flux correlation also show a degree of scatter, despite the higher cadence of X-ray flux points provided by the

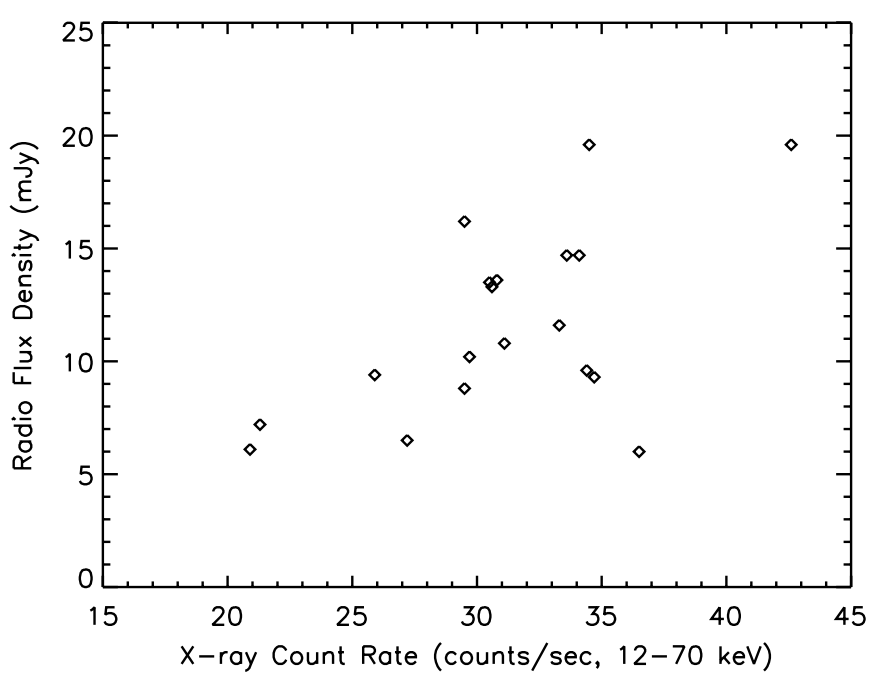

Figure 5. Radio flux density vs. X-ray count rate, for the $20 \mathrm{AMI}$ observations most immediately following the $20 \mathrm{X}$-ray observations we made with Suzaku. Errors on the radio flux density and X-ray count rate are similar to the size of the plotting symbols used in the figure. These model-independent quantities are positively correlated despite the obvious scatter: a Spearman's rank correlation gives $\rho=0.455$, and false correlation probability of 0.044 .

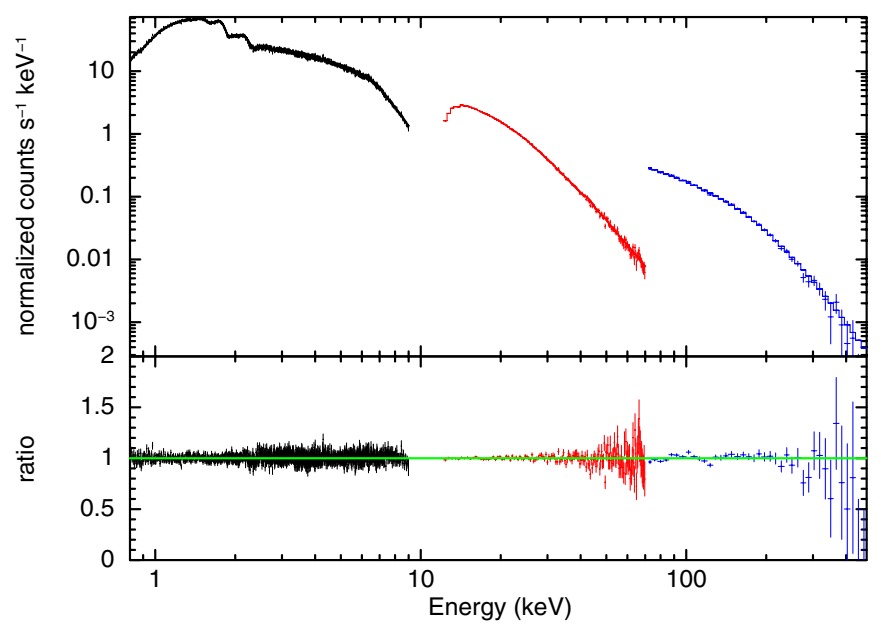

Figure 6. Spectra obtained on 2009 April 14, fitted with a phenomenological model. Warm ionization was modeled using an XSTAR grid, the disk continuum was modeled using the simple "diskpbb" model, and the hard emission was modeled using a broken and cutoff power law. The hard continuum approximates a reflection spectrum. The Fe $\mathrm{K}$ band was modeled using a narrow Gaussian emission line at $6.40 \mathrm{keV}$, and a relativistic Laor emission line; Fe XXV and XXVI absorption lines from the XSTAR grid also affected the Fe $\mathrm{K}$ band. The overall fit is good and all of the parameters are well constrained.

(A color version of this figure is available in the online journal.)

$R X T E / A S M$. This suggests that the scatter in Figure 5 is not exclusively due to a lack of strict simultaneity.

\subsection{Basic Results}

The results detailed in Tables 3 and 4 are generally good fits to the spectra, with $\chi^{2} / v \simeq 1$. Figure 6 depicts a typical fit to the broadband spectrum with the phenomenological model. Figures 7 and 8 show the amplitude of the broad and narrow Fe $\mathrm{K}$ lines, when the data are fitted with a phenomenological continuum. Figure 9 shows a typical fit to the broadband spectrum of Cygnus X-1 with the more physical relativistically blurred disk reflection model.

Where the fits are not formally acceptable, there are likely three main causes. First, calibration uncertainties produced 
Table 3

\begin{tabular}{|c|c|c|c|c|c|c|c|c|c|c|c|c|c|c|c|c|c|c|}
\hline Date & $\chi^{2}$ & $\begin{array}{c}N_{\mathrm{H}} / 10^{21} \\
\left(\mathrm{~cm}^{-2}\right)\end{array}$ & $\begin{array}{c}\mathrm{kT} \\
(\mathrm{keV})\end{array}$ & $\begin{array}{l}K_{\text {disk }} \\
\left(10^{5}\right)\end{array}$ & $\begin{array}{c}R_{\text {disk }} \\
\left(G M / c^{2}\right)\end{array}$ & $\Gamma_{1}$ & $\overline{\Gamma_{2}}$ & $\begin{array}{l}E_{\text {break }} \\
(\mathrm{keV})\end{array}$ & $K_{\mathrm{PL}}$ & $\begin{array}{c}E_{\text {cut }} \\
(\mathrm{keV})\end{array}$ & $\begin{array}{c}E_{\text {fold }} \\
(\mathrm{keV})\end{array}$ & $\begin{array}{l}E_{\text {Laor }} \\
(\mathrm{keV})\end{array}$ & $\begin{array}{c}R_{\text {line }} \\
\left(G M / c^{2} \mathrm{i}\right)\end{array}$ & $\begin{array}{c}K_{\text {Laor }} \\
\left(10^{-2}\right)\end{array}$ & $\begin{array}{l}\text { EW } \\
(\mathrm{eV})\end{array}$ & $\begin{array}{c}0.8-10.0 \\
\left(10^{-8}\right)\end{array}$ & $\begin{array}{c}0.8-500 \\
\left(10^{-8}\right)\end{array}$ & $\begin{array}{c}\text { Disk } \\
\left(10^{-8}\right)\end{array}$ \\
\hline Apr 3 & 2430.2 & $1.3(3)$ & $0.198(3)$ & $1.5(1)$ & $3.3(2)$ & $1.76(1)$ & $1.40(1)$ & $6.4(1)$ & $2.33(3)$ & $22(1)$ & $153(3)$ & $6.70^{+0.01}$ & $4.6(6)$ & $1.5(2)$ & 176.0 & 1.40 & 3.38 & 0.135 \\
\hline Apr 8 & 2282.8 & $1.0^{+0.1}$ & $0.195(3)$ & $1.35(7)$ & $3.1(2)$ & $1.776(5)$ & $1.40(1)$ & 7.1(1) & $1.90(1)$ & $24(1)$ & $163(4)$ & $6.90(4)$ & $3.7(4)$ & $1.3(2)$ & 210.0 & 1.11 & 2.62 & 0.107 \\
\hline Apr 14 & 2455.0 & $1.3(3)$ & $0.191(2)$ & $1.2(1)$ & $2.9(3)$ & $1.763(7)$ & $1.41(1)$ & $7.2(2)$ & $1.58(1)$ & $24(1)$ & $160(4)$ & $6.89(3)$ & $3.6(4)$ & $1.1(1)$ & 217.0 & 0.93 & 2.20 & 0.082 \\
\hline Apr 23 & 2701.8 & $12(2)$ & $0.180(5)$ & $0.8(1)$ & $2.4(4)$ & $1.683(8)$ & $1.41(1)$ & $7.3(5)$ & $1.53(1)$ & $23(1)$ & $159(3)$ & $6.96(2)$ & $4(1)$ & $1.1(2)$ & 187.0 & 0.94 & 2.37 & 0.035 \\
\hline Apr 28 & 2397.9 & $5(1)$ & $0.203(4)$ & $1.1(2)$ & $2.8(5)$ & $1.74(1)$ & $1.43(1)$ & $7.9(1)$ & $2.36(3)$ & $24(2)$ & $167(4)$ & $6.94(3)$ & $3.4(3)$ & $1.4(2)$ & 150.0 & 1.12 & 2.53 & 0.137 \\
\hline May 6 & 2492.4 & $1.0^{+0.1}$ & $0.203(2)$ & $1.60(6)$ & $3.3(1)$ & $1.853(3)$ & $1.42(1)$ & $6.8(1)$ & $2.33(1)$ & $23(1)$ & $152(3)$ & $6.92(3)$ & $3.7(2)$ & $1.6(1)$ & 252.0 & 1.31 & 2.89 & 0.165 \\
\hline May 20 & 2369.2 & $2.0(1)$ & $0.204(2)$ & $1.80(8)$ & $3.5(2)$ & $1.892(8)$ & $1.47(1)$ & $6.6(1)$ & $2.29(2)$ & $22(1)$ & $169(4)$ & $6.89(3)$ & $3.3(3)$ & $1.5(1)$ & 264.0 & 1.26 & 2.63 & 0.185 \\
\hline May 25 & 2507.5 & $3.4(8)$ & $0.209(2)$ & $2.30(7)$ & $4.0(1)$ & $1.965(7)$ & $1.45(1)$ & $6.5(1)$ & $3.20(2)$ & $21(1)$ & $136(3)$ & $6.89(3)$ & $3.4(2)$ & $2.2(2)$ & 300.0 & 1.69 & 3.41 & 0.288 \\
\hline May 29 & 3075.9 & $1.0^{+0.1}$ & $0.210(1)$ & $3.49(7)$ & $4.9(1)$ & $2.121(2)$ & $1.52(1)$ & $6.0(1)$ & $3.95(1)$ & $23(1)$ & $129(2)$ & $6.8(1)$ & $3.4(1)$ & $2.4(1)$ & 340.0 & 1.92 & 3.43 & 0.442 \\
\hline Jun 2 & 2617.4 & $2(1)$ & $0.217(3)$ & $2.7(2)$ & $4.3(3)$ & $2.10(1)$ & $1.56(1)$ & $6.4(1)$ & $4.00(4)$ & $22(1)$ & $136(3)$ & $6.88(5)$ & $3.3(3)$ & $2.2(2)$ & 318.0 & 1.95 & 3.40 & 0.426 \\
\hline Jun 4 & 2659.8 & $2.6(6)$ & $0.214(3)$ & $2.6(1)$ & $4.3(2)$ & $2.135(5)$ & $1.60(1)$ & $5.1(1)$ & $3.89(3)$ & $31(1)$ & $165(5)$ & $6.73(3)$ & $3.0(3)$ & $2.3(2)$ & 308.0 & 1.82 & 3.29 & 0.368 \\
\hline Oct 21 & 2373.6 & $1.1(1)$ & $0.199(2)$ & $1.48(8)$ & $3.2(2)$ & $1.839(5)$ & $1.44(1)$ & $6.6(1)$ & $1.74(1)$ & $22(1)$ & $160(4)$ & $6.8(1)$ & $4.0(3)$ & $1.0(1)$ & 196.0 & 1.00 & 2.20 & 0.130 \\
\hline Oct 26 & 2429.8 & $1.4(4)$ & $0.193(3)$ & $1.29(7)$ & $3.0(2)$ & $1.802(5)$ & $1.42(1)$ & $6.8(1)$ & $1.67(1)$ & $22(1)$ & $172(4)$ & $6.86(4)$ & $3.8(3)$ & $1.0(1)$ & 206.0 & 0.96 & 2.21 & 0.093 \\
\hline Nov 3 & 1969.4 & $6(2)$ & $0.180(7)$ & 1.1(3) & $2.7(8)$ & $1.67(1)$ & $1.41(1)$ & $8.6(8)$ & $1.16(2)$ & $23(1)$ & $172(6)$ & $6.8(1)$ & $3(1)$ & $0.5(1)$ & 100.0 & 0.72 & 1.78 & 0.046 \\
\hline Nov 10 & 2559.7 & $2(1)$ & $0.132(6)$ & $3.8(7)$ & $5.2(9)$ & $1.620(4)$ & $1.41(1)$ & $9.3(5)$ & $0.95(2)$ & $24(1)$ & $170(4)$ & $6.73(3)$ & $1.6(3)$ & $0.4(1)$ & 86.0 & 0.61 & 1.57 & 0.015 \\
\hline Nov 17 & 2407.6 & $3(1)$ & $0.208(3)$ & $1.00(9)$ & $2.6(2)$ & $1.782(6)$ & $1.39(1)$ & $7.0(1)$ & $2.49(2)$ & $24(1)$ & $153(3)$ & $6.92(4)$ & $3.8(4)$ & $1.7(1)$ & 214.0 & 1.44 & 3.43 & 0.124 \\
\hline Nov 24 & 2301.9 & $1.5(5)$ & $0.206(2)$ & $1.35(9)$ & $3.1(2)$ & $1.84(1)$ & $1.45(1)$ & $6.5(2)$ & $2.09(2)$ & $23(1)$ & $175(3)$ & $6.87(4)$ & $3.4(3)$ & $1.4(1)$ & 231.0 & 1.19 & 2.18 & 0.156 \\
\hline Dec 1 & 2335.9 & $1.1(1)$ & $0.200(2)$ & $1.3(4)$ & $3.0(9)$ & $1.795(7)$ & $1.42(1)$ & $6.7(2)$ & $1.88(2)$ & $23(1)$ & $166(4)$ & $6.93(3)$ & $4.4(2)$ & $1.1(1)$ & 192.0 & 1.11 & 2.54 & 0.128 \\
\hline $\operatorname{Dec} 8$ & 2680.6 & $10(3)$ & $0.192(7)$ & $0.9(3)$ & $2.5(6)$ & $1.70(1)$ & $1.41(1)$ & $8.7(8)$ & $1.75(2)$ & $23(1)$ & $170(4)$ & $6.94(3)$ & $3.4(6)$ & $1.1(2)$ & 182.0 & 1.06 & 2.56 & 0.058 \\
\hline Dec 17 & 2566.1 & $5(2)$ & $0.220(4)$ & $1.36(9)$ & $3.1(2)$ & $1.94(1)$ & $1.43(1)$ & $5.9(1)$ & $3.94(4)$ & $24(1)$ & $153(5)$ & $6.90(5)$ & $3.1(2)$ & $3.3(2)$ & 331.0 & 2.07 & 4.48 & 0.274 \\
\hline
\end{tabular}

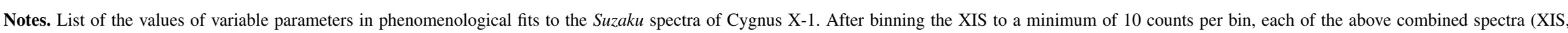

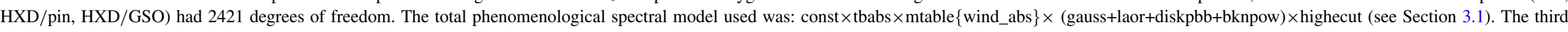

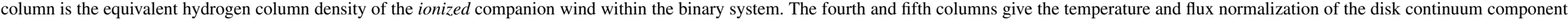

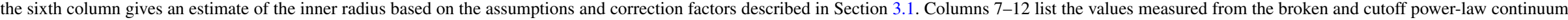

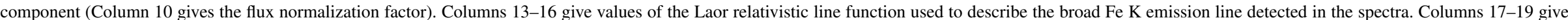

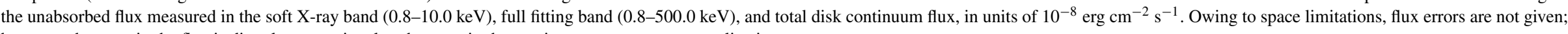
however, the error in the flux is directly proportional to the error in the continuum component normalizations. 
Table 4

Relativistic X-ray Disk Reflection Spectral Parameters

\begin{tabular}{|c|c|c|c|c|c|c|c|c|c|c|c|c|c|c|c|c|}
\hline Date & $\chi^{2}$ & $v$ & $\begin{array}{c}R_{\text {refl }} \\
\left(G M / c^{2}\right)\end{array}$ & $\begin{array}{c}\xi \\
\left(10^{3}\right)\end{array}$ & $\begin{array}{c}K_{\text {refl. }} \\
\left(10^{-5}\right)\end{array}$ & $\begin{array}{c}k T \\
(\mathrm{keV})\end{array}$ & $\begin{array}{l}K_{\text {disk }} \\
\left(10^{5}\right)\end{array}$ & $\begin{array}{c}R_{\text {disk }} \\
\left(G M / c^{2}\right)\end{array}$ & $\Gamma$ & $K_{\mathrm{PL}}$ & $\begin{array}{c}E_{\text {cut }} \\
(\mathrm{keV})\end{array}$ & $\begin{array}{c}E_{\text {fold }} \\
(\mathrm{keV})\end{array}$ & $\begin{array}{c}0.8-500.0 \\
\left(10^{-8}\right)\end{array}$ & $\begin{array}{c}\text { disk } \\
\left(10^{-8}\right)\end{array}$ & $\begin{array}{c}\mathrm{PL} \\
\left(10^{-8}\right)\end{array}$ & $\begin{array}{c}\text { Refl. } \\
\left(10^{-8}\right)\end{array}$ \\
\hline Apr 3 & 2729.5 & 2424 & $1.2(2)$ & $3.0(3)$ & $1.6(1)$ & $0.185(2)$ & $2.0(1)$ & $3.7(2)$ & $1.40(1)$ & $0.58(2)$ & $100(3)$ & $182(9)$ & 6.24 & 0.11 & 4.06 & 2.07 \\
\hline Apr 8 & 2484.1 & 2424 & $1.6(3)$ & $3.0(2)$ & $1.5(1)$ & $0.177(2)$ & $2.1(2)$ & $3.8(2)$ & $1.40(1)$ & $0.40(1)$ & $102(4)$ & $210(10)$ & 4.88 & 0.08 & 3.00 & 1.80 \\
\hline Apr 14 & 2592.0 & 2424 & $1.5(3)$ & $3.0(4)$ & $1.2(1)$ & $0.172(4)$ & $2.1(2)$ & $3.8(2)$ & $1.40(6)$ & $0.36(2)$ & $100(4)$ & $195(10)$ & 4.17 & 0.06 & 2.65 & 1.45 \\
\hline Apr 23 & 2794.8 & 2424 & $1.8(3)$ & $1.6(2)$ & $1.5(2)$ & $0.145(7)$ & $2.2(5)$ & $3.9(3)$ & $1.49(1)$ & $0.79(3)$ & $95(4)$ & $190(10)$ & 4.78 & 0.02 & 3.91 & 0.86 \\
\hline Apr 28 & 2527.4 & 2424 & $1.6(2)$ & $3.3(4)$ & $1.2(2)$ & $0.177(3)$ & $1.6(2)$ & $3.3(4)$ & $1.40^{+0.02}$ & $0.42(3)$ & $96(4)$ & $215(20)$ & 4.69 & 0.06 & 2.98 & 1.65 \\
\hline May 6 & 2675.5 & 2424 & 20(9) & $3.8(4)$ & $1.3(1)$ & $0.183(2)$ & $2.7(2)$ & $4.3(1)$ & $1.47(7)$ & $0.30(3)$ & $147(10)$ & $236(30)$ & 4.45 & 0.14 & 1.81 & 2.55 \\
\hline May 20 & 2500.3 & 2424 & $9(6)$ & $5.0(4)$ & $1.1(1)$ & $0.190(2)$ & $2.3(2)$ & $4.0(1)$ & $1.40^{+0.07}$ & $0.15(1)$ & $170(10)$ & $244(40)$ & 4.00 & 0.17 & 1.26 & 2.57 \\
\hline May 25 & 2069.9 & 1878 & $8(2)$ & $3.8(3)$ & $1.8(1)$ & $0.220(4)$ & $1.9(1)$ & $3.6(1)$ & $1.400^{+0.03}$ & $0.10(1)$ & $162(10)$ & $174(40)$ & 4.85 & 0.29 & 1.16 & 3.40 \\
\hline May 29 & 2444.5 & 1878 & $1.6(2)$ & $4.4(3)$ & $1.7(1)$ & $0.237(3)$ & $1.8(2)$ & $3.5(1)$ & $1.44(2)$ & $0.155(5)$ & $174(10)$ & $94(20)$ & 4.60 & 0.57 & 0.96 & 3.07 \\
\hline Jun 2 & 2012.0 & 1878 & $1.4(1)$ & $4.0(3)$ & $1.8(1)$ & $0.240(2)$ & $1.4(1)$ & $3.1(4)$ & $1.41(1)$ & $0.20(2)$ & $155(10)$ & $169(30)$ & 4.69 & 0.56 & 1.14 & 3.00 \\
\hline Jun 4 & 2149.7 & 1878 & $1.4(2)$ & $4.4(4)$ & $1.6(1)$ & $0.230(2)$ & $1.3(1)$ & $3.0(2)$ & $1.41(1)$ & $0.17(2)$ & $157(20)$ & $165(40)$ & 4.69 & 0.49 & 1.24 & 3.00 \\
\hline Oct 21 & 2537.8 & 2424 & $1.2(2)$ & $4.2(3)$ & $0.81(7)$ & $0.182(2)$ & $2.2(2)$ & $3.9(1)$ & $1.41(1)$ & $0.19(2)$ & $148(10)$ & $185(30)$ & 3.44 & 0.11 & 1.42 & 1.90 \\
\hline Oct 26 & 2653.0 & 2424 & 4(2) & $3.8(4)$ & $1.0(1)$ & $0.169(3)$ & $2.3(2)$ & $4.0(1)$ & $1.41(1)$ & $0.27(2)$ & $130(5)$ & $197(10)$ & 3.87 & 0.07 & 2.05 & 1.72 \\
\hline Nov 3 & 1981.9 & 2424 & $1.8(5)$ & $2.2(3)$ & $1.4(3)$ & $0.14(3)$ & $2.3(2)$ & $4.0(6)$ & $1.45(2)$ & $0.34(5)$ & 109(9) & $240(30)$ & 3.29 & 0.02 & 2.10 & 1.16 \\
\hline Nov 10 & 2831.7 & 2424 & $1.5(1)$ & $3.8(8)$ & $0.30(5)$ & $0.1321)$ & $4.5(6)$ & $5.6(9)$ & $1.48(1)$ & $0.57(2)$ & $93.0(6)$ & $205(20)$ & 3.45 & 0.03 & 2.99 & 0.44 \\
\hline Nov 17 & 2618.8 & 2424 & $6(3)$ & $2.3(2)$ & $2.7(1)$ & $0.17(1)$ & $3.0(5)$ & $4.6(1)$ & $1.44(1)$ & $0.40(2)$ & $146(7)$ & $163(20)$ & 5.44 & 0.04 & 2.61 & 2.75 \\
\hline Nov 24 & 2407.2 & 2424 & $5(2)$ & $3.9(2)$ & $1.2(1)$ & $0.190(2)$ & $1.8(2)$ & $3.5(2)$ & $1.41(1)$ & $0.22(2)$ & $153(7)$ & $180(20)$ & 4.17 & 0.13 & 1.77 & 2.28 \\
\hline Dec 1 & 2316.5 & 2147 & $13(6)$ & $3.1(3)$ & $1.2(1)$ & $0.179(2)$ & $2.6(2)$ & $4.3(7)$ & $1.46(1)$ & $0.35(2)$ & $146(7)$ & $165(20)$ & 4.10 & 0.10 & 2.15 & 1.85 \\
\hline $\operatorname{Dec} 8$ & 2762.6 & 2424 & $2.1(3)$ & $3.3(3)$ & $1.1(2)$ & $0.14(1)$ & $3(1)$ & $4.8(3)$ & $1.42(1)$ & $0.50(2)$ & $109(8)$ & 192(20) & 5.00 & 0.03 & 3.37 & 1.60 \\
\hline Dec 17 & 2332.0 & 2147 & $12(6)$ & $3.5(1)$ & $2.4(1)$ & $0.211(3)$ & $1.8(9)$ & $3.5(2)$ & $1.40^{+0.01}$ & $0.27(2)$ & $154(20)$ & $228(70)$ & 6.68 & 0.25 & 2.23 & 4.19 \\
\hline
\end{tabular}

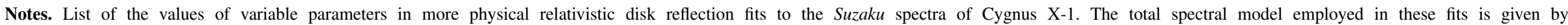

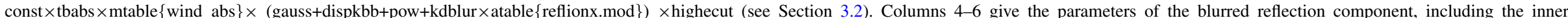

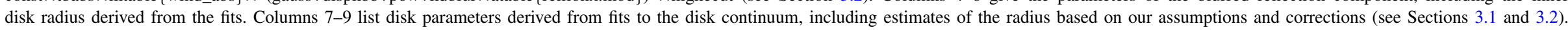

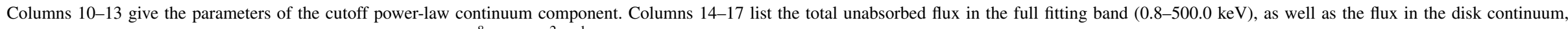

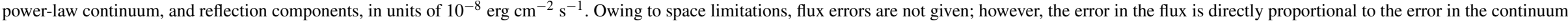
component normalizations. 


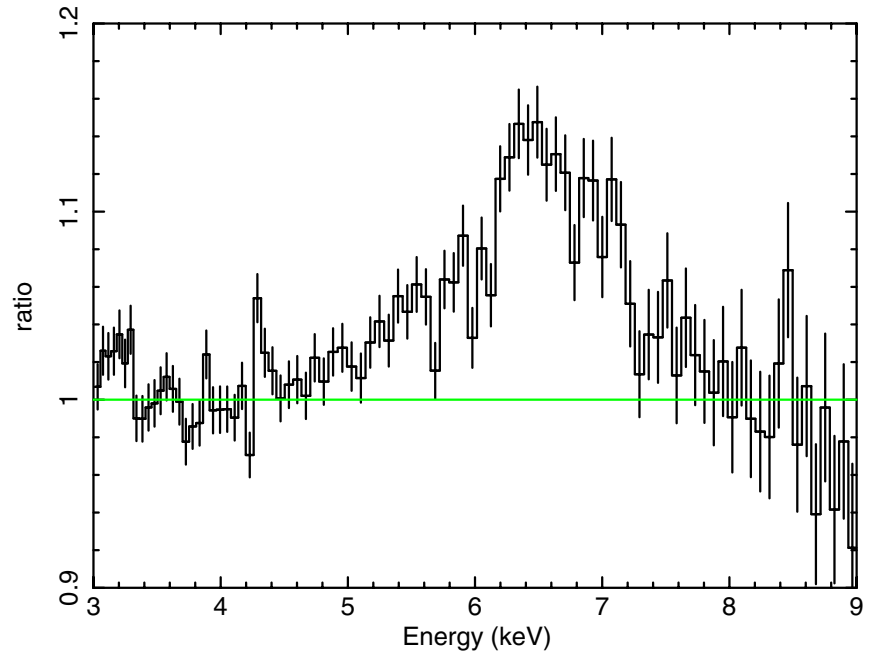

Figure 7. Data/model ratio obtained when the flux of line components affecting the $\mathrm{Fe} \mathrm{K}$ band is set to zero in the best-fit phenomenological model to spectra obtained on 2009 April 14. The narrow neutral Fe K line at $6.4 \mathrm{keV}$ is clearly evident; ionized absorption in the $\mathrm{Fe} \mathrm{K}$ band is not very important in this particular spectrum.

(A color version of this figure is available in the online journal.)

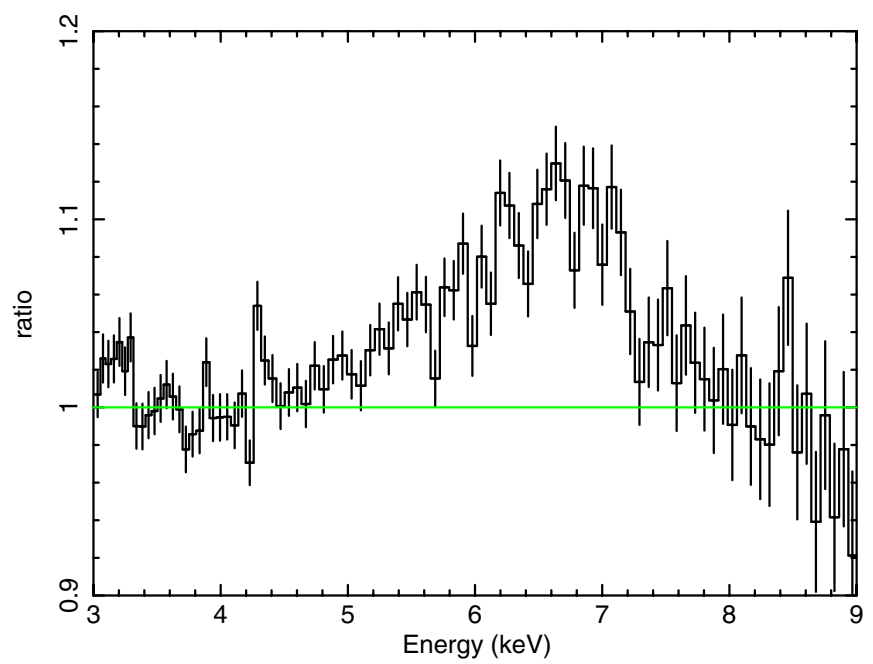

Figure 8. Data/model ratio obtained when the Laor relativistic line component flux is set to zero in the best-fit phenomenological model to spectra obtained on 2009 April 14. Narrow emission and absorption components have been fitted and only the relativistic component to emission in the Fe $\mathrm{K}$ band is shown. The spectrum was binned for visual clarity.

(A color version of this figure is available in the online journal.)

especially poor fits to the spectra obtained on certain days, for instance on May 29. Second, the "reflionx" reflection model has a hard lower bound of $\Gamma=1.4$ for the power-law index of the hard emission assumed to illuminate the disk. A number of fits pegged at this limit, suggesting that a slightly harder power-law might have been favored by the data. Last, the hard emission may be better described using a broken power law, and "reflionx" only assumes a simple power law at low energy. It is notable that most spectra are better fitted with the phenomenological model than with the blurred reflection model. Other efforts have also found that physical models do not provide better fits to spectra from Cygnus X-1 than phenomenological models (Wilms et al. 2006; Nowak et al. 2011). This is likely the result of the greater freedom afforded by a broken power-law and a separate line component, and the limitations of "reflionx" noted above.

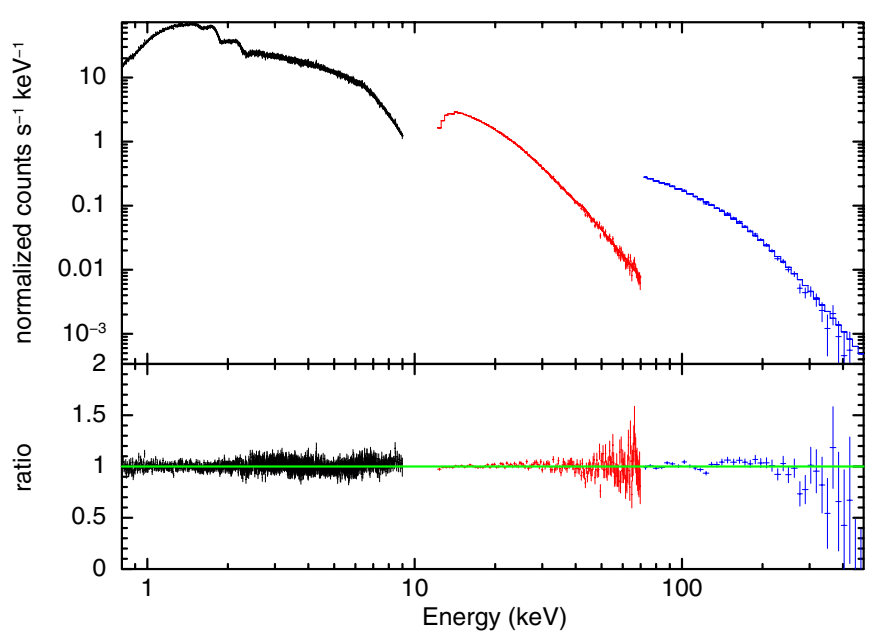

Figure 9. Spectra obtained on 2009 April 14, fitted with a physical blurred disk reflection model. Both dynamical and scattering broadening were included among the effects that shape the broad Fe $\mathrm{K}$ line. Warm ionization was modeled using an XSTAR grid, the disk continuum was modeled using the simple "diskpbb" model. The overall fit is good and all of the parameters are well constrained.

(A color version of this figure is available in the online journal.)

Overall, the quality of the spectral fits-both phenomenological and physical-is such that the resulting measurements and correlations are meaningful.

Given the complexities of these observations, including the use of the HXD pointing position for the XIS, the spacecraft wobble, attempts to correct for the wobble, the use of window and burst options, efforts to mitigate photon pile-up in the XIS, and evolution of the HXD performance, it is not clear what the detector cross-normalization factors (captured by the "constants" in both families of spectral models) should be. The use of constant factors to normalize across different detectors when fitting over a broad energy range is well established (see, e.g., Sobczak et al. 2000, concerning multiple spectra of XTE J1550-564 obtained with RXTE). Moreover, some of the relevant effects likely change between observations. The constant factors were therefore allowed to float in each observation. Values for the constant fell in the $0.90-1.10$ range in the phenomenological fits, and in the 1.02-1.32 range for the blurred reflection fits (values for the PIN/GSO factor fell in the 0.75-1.05 range and 0.80-1.17 range, respectively). A similar observational set-up was used to observe the black hole candidate MAXI J1836-194 using Suzaku (Reis et al. 2012). The values of the cross-normalization factors found in our fits are broadly consistent with values covered by the error contours derived from fits to the spectra of MAXI J1836-194. Small changes to the factors we recorded (e.g., 0.01) cause small changes in the continuum fits, not the line properties. Thus, although uncertainty in the flux offsets could be assessed as a systematic error, it is not one that has a strong effect on the features of interest. Larger changes (e.g., 0.1), or setting the XIS/PIN factor to a value of 1.17 (common in more standard modes), results in dramatically worse fits in many cases, such that no phenomenological nor physical spectral model is acceptable.

In every sense, the phenomenological X-ray spectral fitting results are typical of Cygnus X-1 in the "low/hard" state. The power-law index above the spectral break is found to be quite hard, generally in the range of $1.4 \leqslant \Gamma_{2} \leqslant 1.5$. Similar indices are measured via fits with the blurred "reflionx" model. The 
cutoff energy measured in the phenomenological fits is generally consistent with $k T=23 \mathrm{keV}$. This could be indicative of the electron temperature of the hard X-ray corona, but it is likely that much of the curvature fitted by this low-energy cutoff is actually due to disk reflection. This possibility is supported by the fact that $\Gamma_{2}$ is found to be close to the simple power law in the reflection fits (see Tables 3 and 4), and by the fact that the power-law break energy is generally found to be at or above the $\mathrm{Fe} \mathrm{K}$ line energy, where reflection is expected to produce a similar change in the continuum.

In both families of fits, the temperature of the disk component is found to be quite low, generally at or below $k T \simeq 0.2$ $\mathrm{keV}$. Again, this is fully consistent with black hole X-ray binaries in the "low/hard" state generally, and Cygnus X-1 in particular (see, e.g., Reis et al. 2010; Reynolds \& Miller 2012). The temperature values lie below the energy boundary considered in fits to the XIS spectra; however, simple blackbody functions peak at $2.8 \mathrm{kT}$, and disk blackbody functions peak at a slightly higher energy. Thus, although the accretion disk is found to be cool in all cases, a good deal of its flux falls within the XIS fitting range and serves to constrain the fitting parameters. Disks in this temperature and flux range cannot be detected in observations obtained with the RXTE/PCA and INTEGRAL/JEM-X cameras, for instance, upon which prior coordinated observing campaigns have been built (Wilms et al. 2006).

The inner disk radius implied by the disk continuum is of interest. Tables 3 and 4 give the normalization of the disk component and the radius implied for $d=1.86 \mathrm{kpc}$ (Reid et al. 2011), $M=14.8 M_{\odot}$, and $i=27^{\circ}$ (Orosz et al. 2011), as well as various correction factors. Although a couple of observations imply greater deviations, most normalizations differ by less than a factor of $\leqslant 2$, which would correspond to radii generally varying by less than $40 \%$. It is immediately apparent that this modest variation is far less than the factor of $\sim 4$ variation seen in the radio flux in Figure 4. The normalizations of the disk components are generally higher in the relativistic reflection fits $\left(r_{\text {mean }}=3.9 G M / c^{2}, \sigma_{r}=0.6 G M / c^{2}\right)$ than in the phenomenological fits $\left(r_{\text {mean }}=3.4 \mathrm{GM} / \mathrm{c}^{2}, \sigma_{r}=0.8 \mathrm{GM} / \mathrm{c}^{2}\right)$, suggesting that a small part of the putative disk continuum could potentially be due to blurred atomic lines from disk reflection. Including all of the relevant correction factors, none of the 40 disk continuum fits implies a radius greater than $6 G M / c^{2}$. Indeed, 33 of 40 disk continuum fits suggest $r_{\text {in }} \leqslant 4 G M / c^{2}$, which implies a spin parameter of $a \geqslant 0.6$.

Fits to the relativistic line and blurred reflection spectrum provide an independent check on the innermost extent of the accretion disk and variations in that radius. Phenomenological fits with a separate Laor line component generally find radii between 3-4 GM/ $c^{2}\left(r_{\text {mean }}=3.5 G M / c^{2}, \sigma_{r}=0.6 G M / c^{2}\right)$, in good agreement with fits to the continuum. All radii inferred using fits with the additive "Laor" line component are found to be significantly below $6 G M / c^{2}$. Radii measured via the blurred reflection fits give a broader range of values $\left(r_{\text {mean }}=\right.$ $4.7 G M / c^{2}, \sigma_{r}=5.2 G M / c^{2}$ ), and less agreement with the disk continuum. A number of fits with the relativistically blurred reflection component imply a radius only marginally larger than $1.2 G M / c^{2}$, corresponding to maximal black hole spin. A small number of fits imply a larger disk radius, e.g., $r=20 \pm 9 \mathrm{GM} / \mathrm{c}^{2}$ on May 6. Observations in which larger radii are found generally represent poor measurements: if only those observations with $\operatorname{error}(r) / r \leqslant 3$ are considered, all radii are below $6 \mathrm{GM} / \mathrm{c}^{2}$ and values approach maximal spin. Indeed, weighting the values by

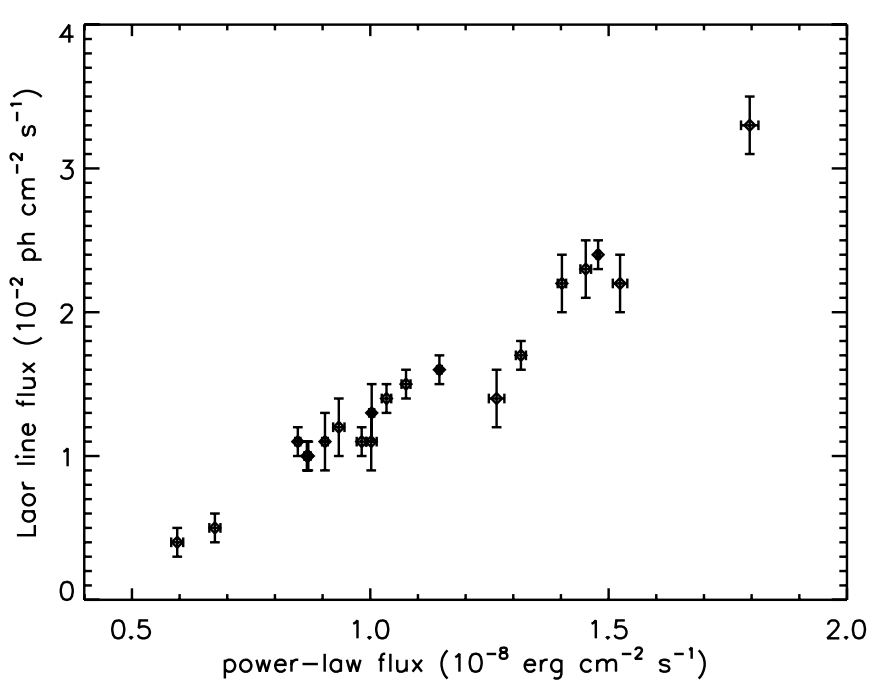

Figure 10. If disk reflection is at work close to black holes, irradiation of the disk by external hard X-ray emission should stimulate an emission line. The flux of the disk line should therefore be driven by, and closely linked to, flux represented by a power-law component. The figure above plots relativistic disk line flux vs. power-law flux for each of the 20 Suzaku observations of Cyg X-1. The Spearman's rank correlation for these two parameters is $\rho=0.958$, and the probability of a false correlation is $3.3 \times 10^{-11}$. The close link between the flux of the relativistic line and hard continuum strongly confirms that reflection from a disk extending close to the black hole is at work in the low/hard state of Cygnus X-1.

the inverse of their errors gives a mean radius of $1.7 \mathrm{GM} / \mathrm{c}^{2}$. Discarding poor measurements, and assuming that the smallest measured values indicate the actual innermost radius defined by the spin of the black hole, the disk continuum and disk reflection fitting results point to a spin in the range $0.6 \leqslant a \leqslant 0.99$.

\subsection{Tests of X-ray Disk Reflection}

This program represents the first time that a CCD spectrometer has observed a single source on 20 occasions, each time achieving the sensitivity needed to detect a relativistic line and disk reflection spectrum. Therefore, some simple checks on the choice of a reflection geometry are very much in order. For instance, simple reflection models predict that the flux of a relativistic line should follow the flux of the power-law continuum that illuminates the disk (e.g., George \& Fabian 1991). Figure 10 shows a tight correlation between the flux in the Laor line component and power-law component in phenomenological fits. A Spearman's rank correlation test gives a coefficient of 0.958 or a probability of just $3.3 \times 10^{-11}$ of a false correlation. This may represent clear support for the illuminated disk geometry assumed by simple disk reflection models.

Evidence of feedback between the disk and corona is predicted to take the form of a relationship between the fraction of the incident flux that is reflected by the disk and the incident power-law photon index (the $R-\Gamma$ correlation; Gilfanov et al. 1999; Zdziarski et al. 2003). Evidence of this correlation has been seen in fits to gas spectrometer data (e.g., Gilfanov et al. 1999; Zdziarski et al. 2003), but this program represents the first opportunity to test the correlation using CCD spectra in which relativistic lines are better revealed and separated from narrow components.

In the case of neutral reflection, the equivalent width of the relativistic line can serve as a proxy for the reflection fraction as the two are closely related (George \& Fabian 1991 predict that $R \sim \mathrm{EW} / 180 \mathrm{eV}$, where $R$ is the reflection fraction). 


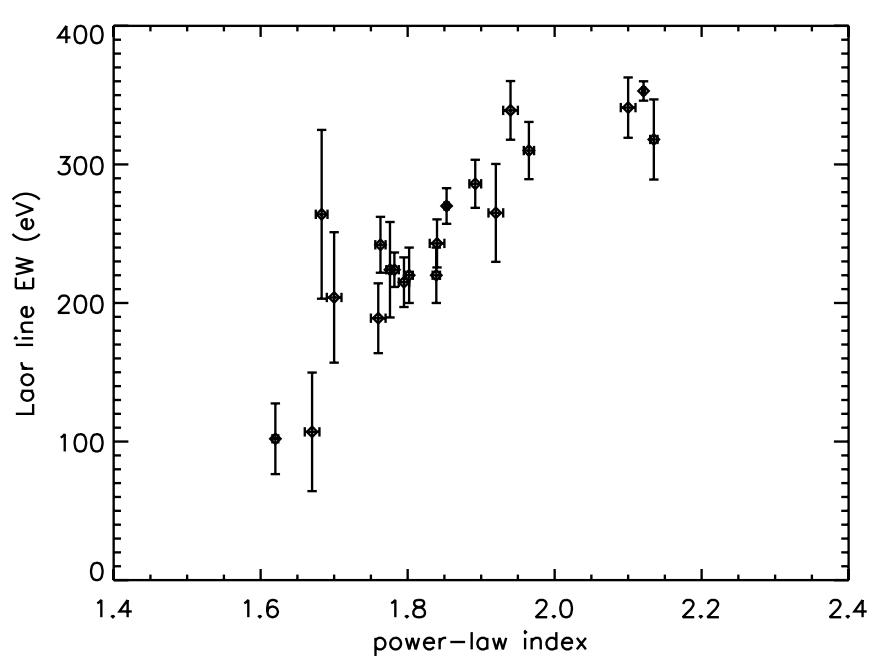

Figure 11. In prior reflection studies, a correlation has been observed between the reflection fraction $(\Omega / 2 \pi)$ vs. the illuminating power-law index; this is taken as evidence of feedback between the corona and disk (Zdziarski et al. 2003). Since the equivalent width of a disk line and the reflection fraction are related for a given ionization (e.g., George \& Fabian 1991), the line equivalent width can be used as a proxy for the reflection fraction. The plot above shows the relativistic Laor line equivalent width vs. the soft X-ray power-law index $\left(\Gamma_{1}\right)$ measured in our phenomenological fits. As expected, the two are tightly correlated. A Spearman's rank correlation test returns a coefficient of $\rho=0.928$, and the probability of a false correlation is $3.9 \times 10^{-9}$.

Figure 11 plots the equivalent width of the Laor line component versus the soft power-law index measured in phenomenological fits to the X-ray spectra. A tight, positive correlation is again observed. A Spearman's rank correlation test gives a coefficient of $\rho=0.921$, with a false correlation probability of just $3.9 \times 10^{-9}$. The slope of this relationship is clearly greater than unity, and broadly consistent with $\simeq 2$. This is broadly consistent with the linear relationship expected between these parameters, and thus broadly consistent with feedback between the disk and corona. The fact that the slope is greater than unity may hint at the need to treat reflection more self-consistently, changes to the disk albedo due to ionization, and/or the influence of gravitational light bending, which predicts a slope steeper than unity if the hard X-ray source is very close to a spinning black hole (Miniutti \& Fabian 2004).

Spectral fits with the relativistically blurred disk reflection spectrum allow the flux in the directly observed power-law and the reflection component to be measured independently and selfconsistently. It is interesting to note that the reflected flux-not the direct power-law flux - is found to be the dominant flux component in half of the observations (see Table 4). Moreover, taking the ratio of the reflected flux to the total flux as a proxy for the reflection fraction $R$, there is no significant correlation between $R$ and $\Gamma$ in the full reflection fits. Together, these results suggest that feedback between the disk and corona, and perhaps the nature of the corona itself, may be more complex than prior studies revealed.

Yet it is difficult to discount the extremely tight correlation between the relativistic line flux and power-law flux (see Figure 10 and the correlations listed in Table 6) based on the phenomenological fits. That correlation is so tight that it calls into doubt the results obtained from the more physical models including a blurred reflection component (see below). There is a simple way in which these apparently contradictory results can be reconciled, however. The power law in the phenomenological fits is a broken power law, meant to mimic a proper reflection spectrum. The flux in this component may not be direct flux, but rather a mixture of direct and reflected flux. Correlating the line and continuum flux would then be a spurious procedure of (at least partly) correlating a component against itself. A very tight relationship should result from such a test. More discussion of the reflection models and their consequences follows in Section 6.

Fits with the relativistically blurred "reflionx" component also suggest a moderately high ionization parameter, generally in the $3<\log (\xi)<4$ range (see Table 4 ). As with the other parameters measured in fits to these $S u z a k u$ observations, this level of ionization is typical of the low/hard state (see, e.g., Reis et al. 2008; Blum et al. 2009). In this regime, He-like and $\mathrm{H}$-like charge states of $\mathrm{Fe}$ are expected to be the most prominent. The Laor line centroid energy values were restricted to the range appropriate for these charge states, but many of the fits measured centroids within this range. Thus, the reflection fits and the separate line fits are in broad agreement with regard to the ionization of the disk.

The details of fits to the narrow Gaussian line fixed at $6.40 \mathrm{keV}$ are omitted from Tables 3 and 4 owing to space limitations, and because this feature is not of central importance. The narrow emission line flux was generally found to be consistent with $1.0 \times 10^{-3}$ photons $\mathrm{cm}^{-2} \mathrm{~s}^{-1}$ and an equivalent width of 15-20 eV. These values are consistent with prior detections of this line component with ASCA (Ebisawa et al. 1996) and at high resolution using the Chandra/HETGS (Miller et al. 2002; Nowak et al. 2011). Moreover, these values are consistent with the illumination of cold clumps in the companion wind or the outer accretion disk (Ebisawa et al. 1996).

\section{CORRELATING X-RAY AND RADIO PARAMETERS}

The results of running Spearman's rank correlation tests on different pairings of radio flux density and X-ray spectral fitting parameters, and between different pairings of X-ray spectral fit parameters only, are given in Tables 5-8. The detailed results of these tests are given below.

\subsection{On Disk Radius and Jet Power}

Figures 12 and 13 plot radio flux density against values of the inner disk radius. Figure 12 plots inner disk radii derived from phenomenological fits, while Figure 13 shows the inner disk radii derived from fits including the relativistically blurred reflection model. In these figures, it is clear that individual measures of the inner disk radius generally lie within a very narrow range of values. It is also clear that variations in the radio flux density occur without changes in the inner disk radius. This lack of a clear correlation is confirmed in rank correlation tests against the line and reflection-derived radii, and the disk normalization from which continuum-based radii derive. There is a single exception that merits additional scrutiny.

In fits including the relativistically blurred reflection model, the inner disk normalization (radius) measured through continuum fits is anti-correlated with radio flux density: $\rho=-0.533$, corresponding to a $1.5 \%$ chance of a false correlation. In this instance, the correlation is skewed by just two data points, derived in fits to the spectra obtained on June 2 and 4 (see Table 4). There is no evidence of a general trend in the normalization (radius) values in question. As noted previously, data taken at that time are particularly affected by calibration problems in the 1.5-3.5 keV band (for a discussion of similar problems, see Nowak et al. 2011). 
Table 5

Rank Correlations of Radio Flux and Phenomenological X-ray Model Parameters

\begin{tabular}{llcc}
\hline \hline Parameter 1 & \multicolumn{1}{c}{ Parameter 2 } & Spearman's $\rho$ & $\begin{array}{c}\text { False Alarm } \\
\text { Probability }\end{array}$ \\
\hline Radio flux density & $\Gamma_{2}$ & 0.647 & 0.002 \\
Radio flux density & Power-law flux & 0.646 & 0.002 \\
& $\quad(0.8-10.0 \mathrm{keV})$ & & \\
Radio flux density & Disk flux & 0.636 & 0.003 \\
Radio flux density & $\Gamma_{1}$ & 0.633 & 0.003 \\
Radio flux density & Disk $k T$ & 0.615 & 0.004 \\
Radio flux density & 0.8-10 keV flux & 0.610 & 0.004 \\
Radio flux density & Laor flux & 0.606 & 0.005 \\
Radio flux density & Power-law norm. & 0.604 & 0.005 \\
Radio flux density & Laor EW & 0.598 & 0.005 \\
Radio flux density & $E_{\text {break }}$ & -0.555 & 0.011 \\
Radio flux density & $0.8-500$ keV flux & 0.491 & 0.028 \\
Radio flux density & Pin count rate & 0.455 & 0.044 \\
Radio flux density & $R_{\text {in }}$ (Laor) & -0.369 & 0.109 \\
Radio flux density & Disk norm. & 0.312 & 0.180 \\
Radio flux density & $E_{\text {fold }}$ & -0.263 & 0.262 \\
Radio flux density & Laor Line Centroid & 0.151 & 0.524 \\
Radio flux density & $E_{\text {cut }}$ & -0.012 & 0.958 \\
\hline
\end{tabular}

Notes. List of the Spearman's rank correlation coefficient, $\rho$, and the associated false correlation probability, for different phenomenological X-ray fitting parameters vs. radio flux density.

Table 6

Rank Correlations of Selected Phenomenological X-ray Model Parameters

\begin{tabular}{llcc}
\hline \hline Parameter 1 & Parameter 2 & Spearman's $\rho$ & False Alarm Probability \\
\hline Power-law flux & Laor flux & 0.958 & $3.3 \times 10^{-11}$ \\
Laor equiv. width & $\Gamma_{1}$ & 0.928 & $3.9 \times 10^{-9}$ \\
Power-law flux & Disk flux & 0.872 & $5.4 \times 10^{-7}$ \\
Disk flux & Laor flux & 0.865 & $8.5 \times 10^{-7}$ \\
Laor equiv. width & $\Gamma_{2}$ & 0.729 & $2.7 \times 10^{-4}$ \\
Disk flux & Disk norm. & 0.655 & 0.002 \\
Disk norm. & Disk $k T$ & 0.452 & 0.045 \\
Power-law norm. & Disk norm. & 0.447 & 0.048 \\
Power-law flux & $R_{\text {in }}$ (Laor) & -0.175 & 0.462 \\
Disk flux & $R_{\text {in }}$ (Laor) & -0.147 & 0.537 \\
\hline
\end{tabular}

Notes. List of the Spearman's rank correlation coefficient, $\rho$, and the associated false correlation probability, for different phenomenological $\mathrm{X}$-ray fitting parameters.

Given that (1) only two points have driven the results of the rank correlation test, (2) those two points are derived in spectra that are less reliable than most other spectra, (3) fits to the disk continuum with a more phenomenological model do not find a significant correlation $(\rho=0.312$, with a $18 \%$ chance of false correlation), (4) only four radius measurements are statistically inconsistent with a very narrow range $\left(3 G M / c^{2} \leqslant\right.$ $\left.r_{\text {in }} \leqslant 4 G M / c^{2}\right)$, (5) systematic errors are likely to be much larger than statistical errors, and (6) independent fits to the Fe K line and reflection spectrum find no correlation with radio flux density, this apparent anti-correlation is not robust.

Taken as a whole, the data show that the jet power (as traced by radio flux) is likely not modulated by changes in the inner radius of the accretion disk in the low/hard state.

\subsection{Other Modes of Disk-Jet Coupling}

Although variations in the inner disk radius do not appear to be the source of fluctuations in the jet, there is evidence of
Table 7

Rank Correlations of Radio Flux and X-ray Reflection Model Parameters

\begin{tabular}{llcc}
\hline \hline Parameter 1 & \multicolumn{1}{c}{ Parameter 2 } & Spearman's $\rho$ & $\begin{array}{c}\text { False Alarm } \\
\text { Probability }\end{array}$ \\
\hline Radio flux density & Disk $k T$ & 0.675 & 0.001 \\
Radio flux density & Disk flux & 0.640 & 0.002 \\
Radio flux density & $E_{\text {cut }}$ & 0.600 & 0.005 \\
Radio flux density & Reflection flux & 0.551 & 0.012 \\
Radio flux density & Disk norm. & -0.533 & 0.015 \\
Radio flux density & Power-law norm. & -0.436 & 0.054 \\
Radio flux density & $\xi$ & 0.415 & 0.069 \\
Radio flux density & Power-law flux & -0.414 & 0.070 \\
Radio flux density & 0.8-500.0 keV flux & 0.357 & 0.122 \\
Radio flux density & Reflection norm. & 0.332 & 0.153 \\
Radio flux density & $E_{\text {fold }}$ & -0.274 & 0.243 \\
Radio flux density & $\Gamma$ & -0.204 & 0.388 \\
Radio flux density & $R_{\text {in }}$ (reflection) & 0.090 & 0.707 \\
\hline
\end{tabular}

Notes. List of the Spearman's rank correlation coefficient, $\rho$, and the associated false correlation probability, for different physically motivated X-ray fitting parameters vs. radio flux density.

Table 8

Rank Correlations of Selected X-ray Reflection Model Parameters

\begin{tabular}{llrc}
\hline \hline Parameter 1 & Parameter 2 & Spearman's $\rho$ & False Alarm Probability \\
\hline Reflection flux & Disk flux & 0.881 & $3.0 \times 10^{-7}$ \\
Disk norm. & Disk $k T$ & -0.795 & $2.8 \times 10^{-5}$ \\
Power-law flux & Reflection flux & -0.662 & 0.002 \\
Disk kT & $\xi$ & 0.658 & 0.002 \\
Reflection flux & $\Gamma$ & -0.340 & 0.142 \\
Disk norm. & $R_{\text {in }}$ & 0.320 & 0.169 \\
Reflection/total flux & $R_{\text {in }}$ & 0.271 & 0.248 \\
Reflection/total flux & $\Gamma$ & -0.254 & 0.280 \\
Reflection flux & $R_{\text {in }}$ & 0.234 & 0.321
\end{tabular}

Notes. List of the Spearman's rank correlation coefficient, $\rho$, and the associated false correlation probability, for different physically motivated X-ray fitting parameters.

disk-jet coupling in our observations. In the phenomenological fits to the X-ray spectra, the disk flux and disk temperature are positively correlated with the radio flux density $(\rho=0.636$ and $\rho=0.615$, respectively, implying $0.3 \%$ and $0.4 \%$ probability of false correlation). In the more physical spectral fits, wherein a relativistically blurred disk reflection component is included, the disk temperature and flux are the two parameters that are most strongly correlated with the radio flux $(\rho=0.675$ and $\rho=0.640$, respectively, implying $0.1 \%$ and $0.2 \%$ probability of false correlation). Figure 14 plots radio flux density versus the disk continuum flux measured in the phenomenological and more physical models, while Figure 15 plots radio flux density versus the disk temperature in each family of models. It is notable that all of these correlations are stronger than the correlation between radio flux and the HXD/pin count rate, which is indicative of the correlations found when lowresolution X-ray monitoring data or gas spectrometer data are used to study disk-jet connections.

Simple accretion disk theory and fundamental work on advection-dominated flows show that the mass accretion rate may serve to modulate the disk temperature, flux, and its inner radial extent (e.g., Esin et al. 1997). Above a critical threshold in mass accretion rate, however, it is likely that the disk is always close to the innermost stable circular orbit. This appears 


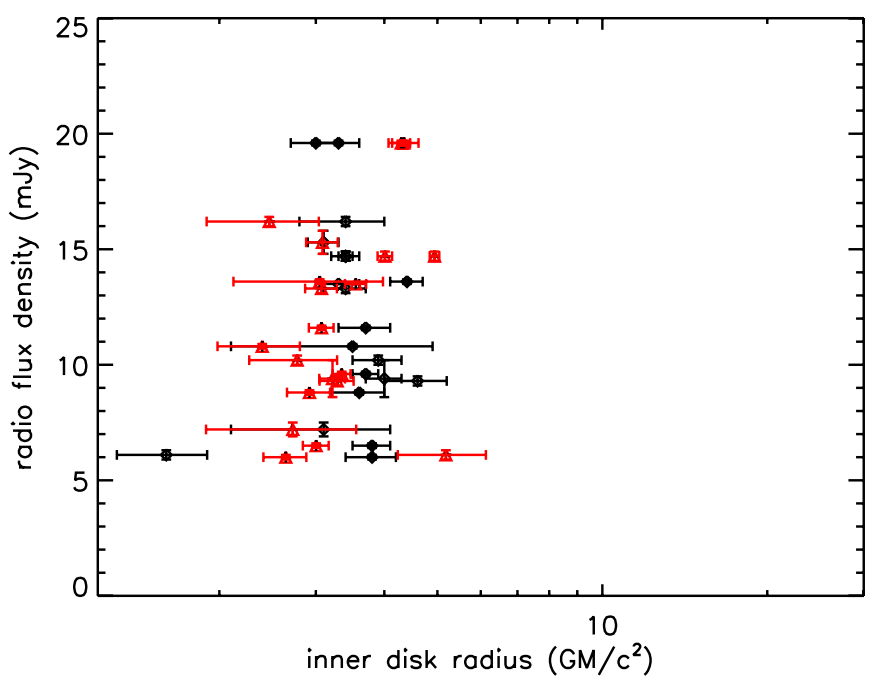

Figure 12. Variations in the inner radius of the accretion disk are one plausible means by which the power in a relativistic jet might be modulated in black hole systems. The figure above plots radio flux density vs. the inner disk radius as measured by fits to the broad $\mathrm{Fe} \mathrm{K}$ line with a Laor line profile (black) and fits to the thermal continuum with a simple disk model (red). Despite strong radio variability, there is clearly little variability in the inner disk radius. The Spearman's rank correlation coefficients with radio flux for these radius estimators are just $\rho=-0.369$ (line) and $\rho=0.312$ (continuum), indicating that radio jet power and inner disk radius are not strongly correlated.

(A color version of this figure is available in the online journal.)

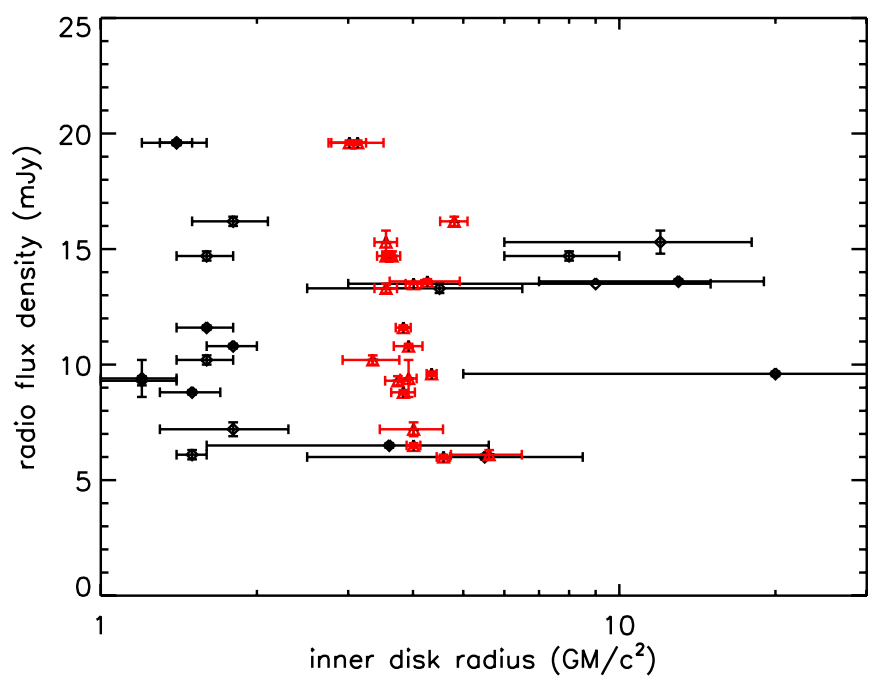

Figure 13. Variations in the inner radius of the accretion disk are a plausible means by which the power in a relativistic jet might be modulated in black hole systems. The figure above plots radio flux density vs. the inner disk radius as measured by fits to the disk reflection spectrum with variable relativistic blurring (black) and fits to the thermal continuum with a simple disk model (red). Despite strong radio variability, there is clearly little variability in the inner disk radius. The Spearman's rank correlation coefficients with radio flux for these radius estimators are just $\rho=0.090$ (line) and $\rho=-0.533$ (continuum). After filtering out two suspect continuum-derived radius estimates (see Section 5.1), neither estimator of the inner disk radius is significantly correlated with the radio flux.

(A color version of this figure is available in the online journal.)

to be largely confirmed by recent observations with $\mathrm{CCD}$ and dispersive X-ray spectrometers, especially after disk correction factors are considered (e.g., Reynolds \& Miller 2012). The critical point seems to be $L / L_{\text {Edd }} \simeq 0.001$, corresponding to $\dot{m}_{\text {Edd }} \simeq 0.01$ for a canonical accretion efficiency of $10 \%$ (Miller

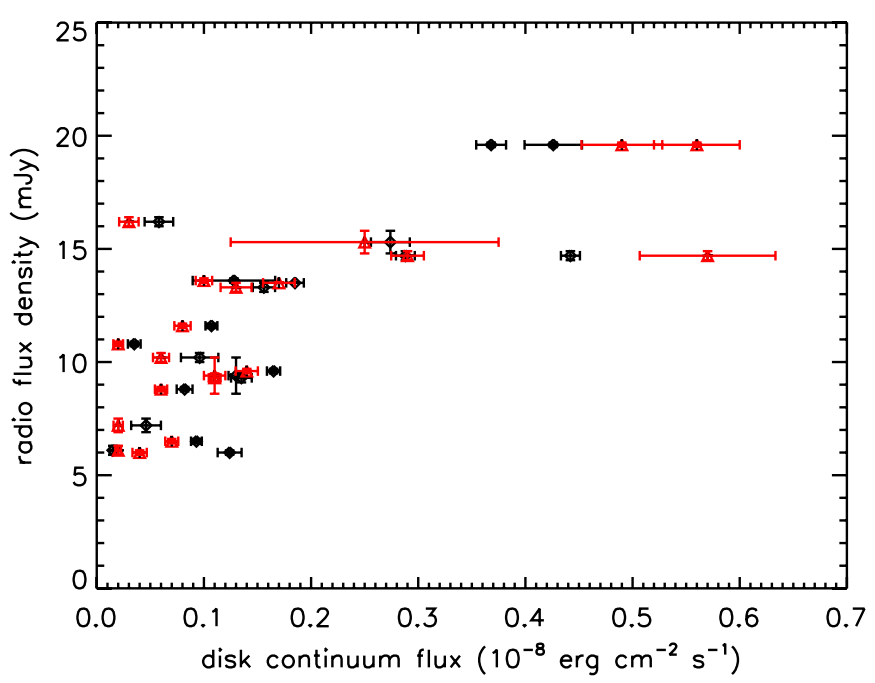

Figure 14. Flux from an accretion disk-especially if the flux traces $\dot{m}$ directly - may serve to modulate jet properties. The plot above shows the radio flux density observed from Cygnus X-1 vs. the unabsorbed $0.8-10.0 \mathrm{keV}$ disk component flux measured in simple fits (black) and blurred reflection fits (red) to Suzaku spectra. Both estimators of the disk continuum flux are positively correlated with radio flux at the $99.7 \%$ level of confidence.

(A color version of this figure is available in the online journal.)

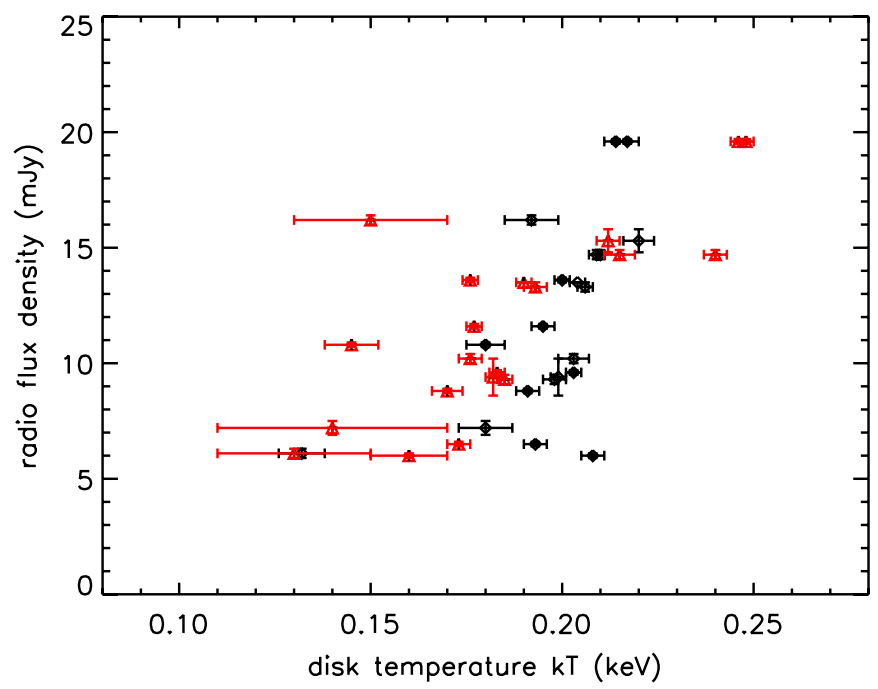

Figure 15. Radio jet flux density vs. inner disk color temperature, based on fits to the disk continuum in both phenomenological (black) and blurred reflection (red) spectral models. As with estimates of the disk continuum flux, both estimates of the inner disk color temperature are positively correlated with radio flux at more than $99.6 \%$ confidence.

(A color version of this figure is available in the online journal.)

et al. 2006a; Rykoff et al. 2007; Tomsick et al. 2009; Reis et al. 2010).

The luminosity of Cygnus X-1 is over this threshold: the mean bolometric luminosity, based on the values in Table 3 , is $L=1.2 \times 10^{37} \mathrm{erg} \mathrm{s}^{-1}$ or $L / L_{\mathrm{Edd}} \simeq 0.006$. Thus, it is possible that variations in the mass accretion rate primarily affect the disk temperature and flux rather than the inner disk radius. This is supported by the relative constancy of the disk radii that we have derived in both phenomenological and more physical spectral models, using both the disk continuum and the disk reflection spectrum (see Figures 12 and 13). The jet in the low/hard state may be sensitive to the only disk parameters that can vary in this regime. It is particularly appealing that the disk flux-a 


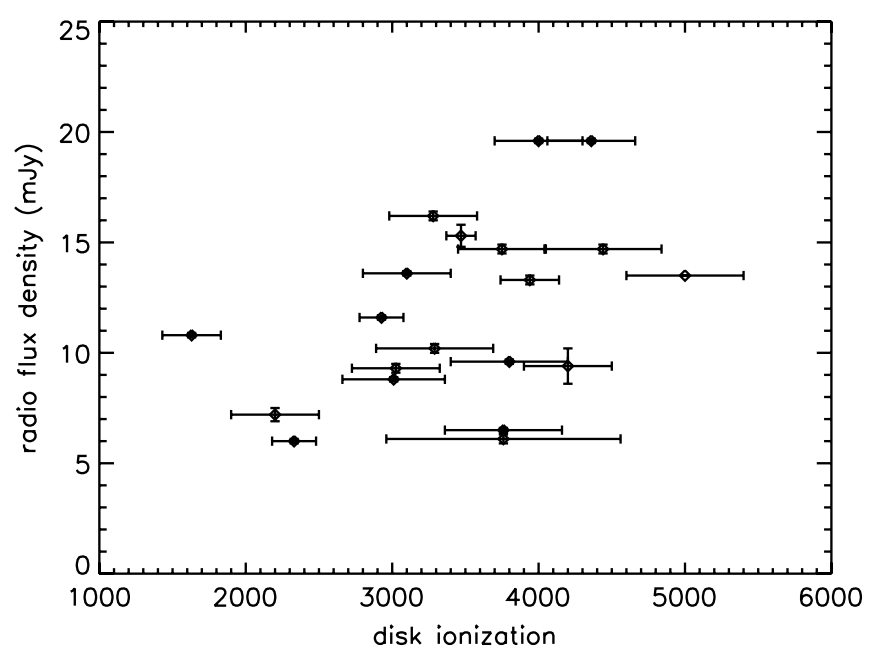

Figure 16. Radio flux density vs. the ionization of the accretion disk, as measured in fits with a relativistically blurred disk reflection model. The general trend seen in this plot is supported by a Spearman's rank correlation test, which returns a coefficient of $\rho=0.415$ (corresponding to a $6.9 \%$ chance of a false correlation). Though tentative, this correlation is as strong as that between radio flux and the hard X-ray count rate $(\rho=0.455$, see Figure 5), and it may suggest that jet production is partly tied to disk ionization. A simple physical explanation might be that poloidal magnetic field lines are more easily anchored in the ionized skin of a disk than in a low-ionization disk with less free charge.

proxy for the mass accretion rate through the disk-is positively correlated with radio flux. This could indicate a simple coupling between the mass accretion rate in the disk and the power of the jet.

It is also interesting to note that the disk ionization (as measured by the reflection model) shows a weak positive correlation with the radio flux density $(\rho=0.415)$, indicating a $6.9 \%$ chance of false correlation. This parameter is difficult to measure, and errors are typically large. Nevertheless, a plot of radio flux density versus the ionization parameter appears to show a weak positive trend (see Figure 16).

Again, prior correlations between radio flux density and X-ray flux from monitoring data are effectively a correlation between the radio flux density and the hard X-ray flux. Those correlations make no statement about the origin of the hard X-ray flux nor how the hard X-ray flux might be divided between different components. Table 5 shows that radio flux density is correlated with the indices and flux of the (broken) power-law component used in phenomenological spectral fits to the Suzaku data. This provides a relatively straightforward basis for the correlation between radio flux density and hard X-ray flux (see Figures 5 and 17).

However, the broken and the cutoff power-law model used in the phenomenological fits is really a proxy for a full reflection model. Correlations using the more physical model parameters give the opposite result: radio flux density is anti-correlated with the direct power-law flux ( $\rho=-0.414$, corresponding to a $7 \%$ chance of false correlation). Instead, the radio flux density is positively correlated with the reflected flux $(\rho=0.551$, giving a $1.2 \%$ chance of false correlation; see Table 7 and Figure 18). In this context, it is also useful to build on the results in Section 4.3 by noting that the reflected flux is found to be anticorrelated with the direct power-law flux $(\rho=-0.662$, giving a $0.2 \%$ chance of false correlation; see Figure 19), in apparent contradiction to simple disk reflection models. As discussed below, this finding is fully consistent with a model involving light bending effects (e.g., Miniutti \& Fabian 2004).

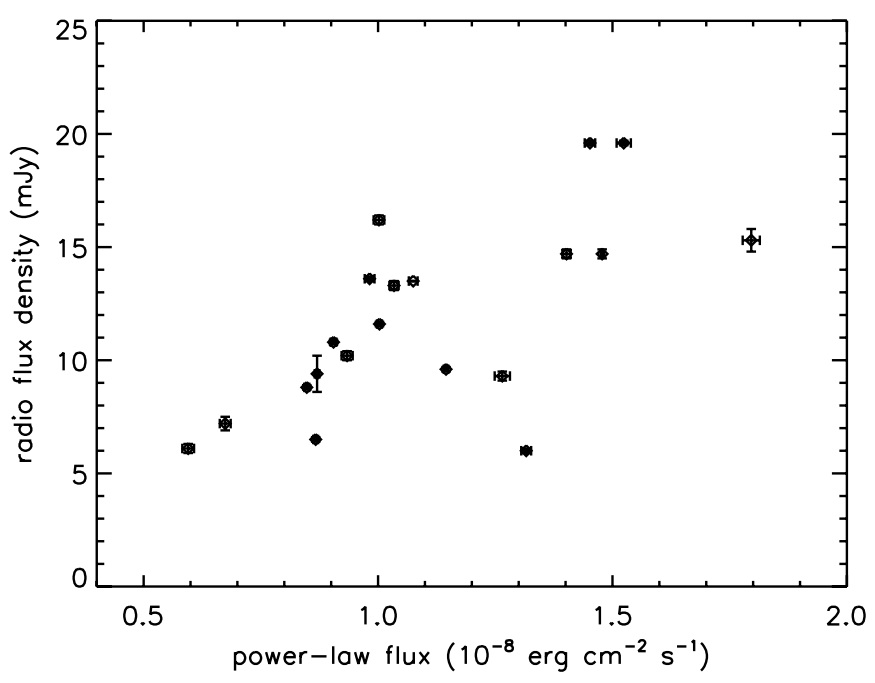

Figure 17. Corona-if traced effectively by a power-law component-may also modulate jet properties in accreting black holes. The plot above shows the radio flux density observed from Cygnus X-1 vs. the unabsorbed $0.8-10.0 \mathrm{keV}$ powerlaw component flux measured in phenomenological fits to the Suzaku spectra. These parameters are positively correlated at the $99.8 \%$ level of confidence. At least in the disk/corona decomposition possible in simple spectral fits, then, both the disk and corona are correlated with radio jet flux, and both may contribute to jet formation and modulation.

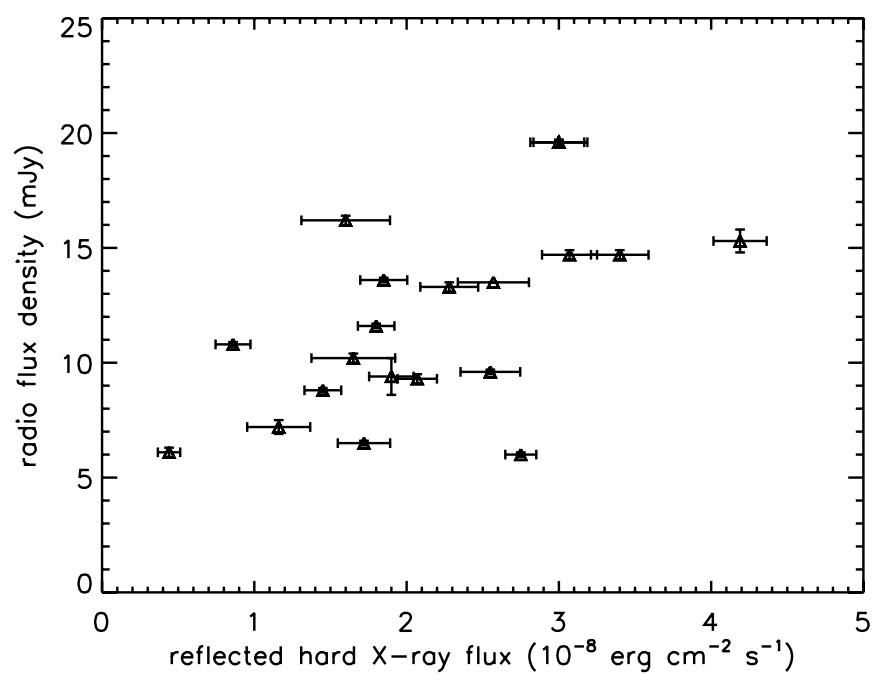

Figure 18. Radio flux density observed from Cygnus X-1 vs. the unabsorbed $0.8-10.0 \mathrm{keV}$ reflected disk flux measured in more physical model fits to the Suzaku spectra. In this X-ray spectral decomposition, the radio flux is anticorrelated with the power-law flux (93\% confidence), and positively correlated with the reflected flux ( $99 \%$ confidence). This result may constitute specific support for a plasma ejection model of the hard X-ray corona, in which radiation pressure from reflected disk flux helps to launch the base of a jet (Beloborodov 1999).

\section{DISCUSSION}

We observed Cygnus X-1 in a standard low/hard state on 20 occasions with Suzaku during 2009, while making frequent monitoring observations with the AMI radio telescope (see Tables 1 and 2). Spectral fits to the X-ray data employed both phenomenological models and more physical disk reflection models (see Tables 3 and 4). Correlations were then calculated between parameters derived in the X-ray spectral fits and the radio fluxes obtained via the monitoring program (see Tables 5-8). This program marks the first time that a mission covering a broad $\mathrm{X}$-ray bandpass, but also offering moderate spectral resolution 


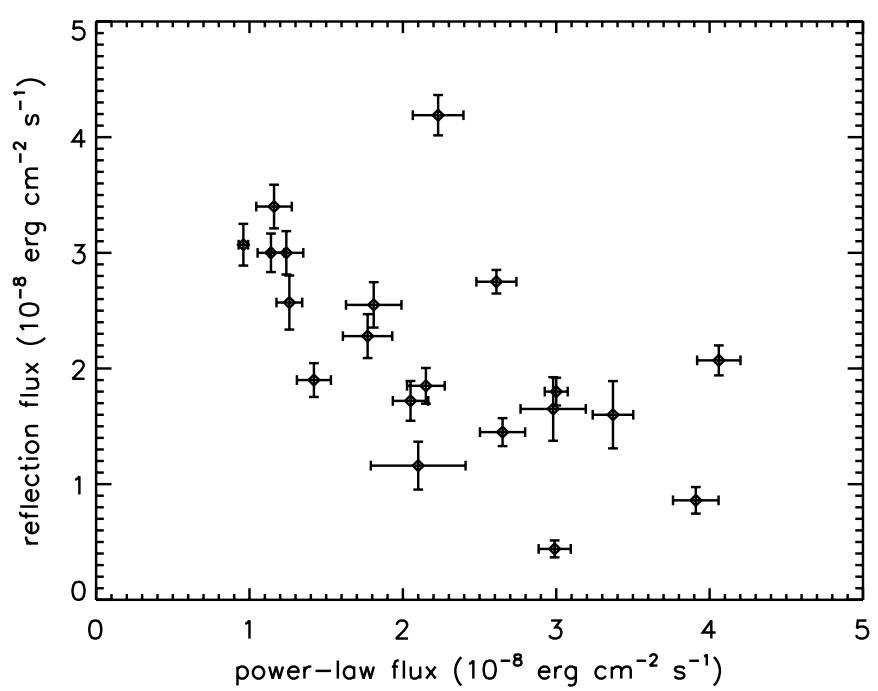

Figure 19. Flux in the blurred disk reflection component vs. the flux in the power-law component. Flux from the power-law component is assumed to be directly observed. In simple reflection models, reflection flux should vary linearly with the power-law flux. However, if the power-law flux originates close to a spinning black hole, gravitational light bending can alter the flux relationship. The anti-correlation shown in the plot above $(\rho=-0.662$, giving a $0.2 \%$ chance of false correlation) is suggestive of a source height that is close to $\simeq 20 \mathrm{GM} / \mathrm{c}^{2}$ according to the calculations of Miniutti \& Fabian (2004).

and low-energy coverage via a CCD spectrometer, has made monitoring observations capable of detecting direct and reflected emission from the cool disk. Numerous measures were taken to ensure the best possible study of the disk, including explicit modeling of the ionized companion wind, the use of an irradiated disk model, and the use of a blurred reflection model suited to high ionization. A number of potential insights into disk-jet coupling, the nature of the hard X-ray corona, and interactions between the disk, corona, and jet are discussed below.

A central result of this program is that variations in power of the relativistic jet (as traced by the radio flux) do not appear to be driven by variations in the inner disk radius, at least not within the confines of the sampling and methods employed in this program. This result is based on the absence of significant correlations between inferred disk radii and radio flux (see Tables 3-6). However, an early indication of this result is already clear from a visual inspection of Figure 4: the radio flux shows much higher fractional variability than the X-ray flux. Given that disk radii depend on the square root of the disk flux normalization, the fractional variability in the disk normalization would have to be very strong in order to match the radio flux variations. The spectral fits reveal only small changes in the disk normalization, indicating only small changes in the disk radius. This result is corroborated via fits to the relativistic iron disk line, and fits to the full disk reflection spectrum, which are completely independent measures of the disk radius. The inner disk radii derived in those fits are also uncorrelated with radio flux, and lie within narrow ranges (see Tables 3, 4, 5, and 7, and Figures 12 and 13).

The constancy of the inner disk in fits to the thermal continuum of LMC X-3 in the high/soft state, for instance, was recently explained in terms of the disk remaining close to the ISCO (Steiner et al. 2010). The relative stability of the inner disk radii derived in our X-ray spectral fits (see Tables 3 and 4, and Figures 12 and 13) is also an indication that the inner disk tends to sit near the innermost stable circular orbit. Indeed, the best fits strongly point to radii consistent with the
ISCO around a spinning black hole, i.e., within $6 G M / c^{2}$. In this regard, Cygnus X-1 is likely not unique. A growing number of results from spectrometers with low-energy coverage suggest that disks may remain at the ISCO in the low/hard state, down to $L / L_{\text {Edd }} \simeq 0.001$ (Miller et al. 2006a; Rykoff et al. 2007; Reis et al. 2010; Reynolds et al. 2010; Reynolds \& Miller 2012; Duro et al. 2011). Observations also indicate that disks may begin to radially recede as per the predictions of ADAF models at or below this threshold (Tomsick et al. 2009). In its low/hard state, Cygnus X-1 is a factor of a few above this critical Eddington fraction, consistent with fitting results suggesting that the disk is close to the ISCO.

Given that a steady, compact jet operates in the low/hard state of Cygnus X-1 while the disk appears to remain close to the ISCO, it is possible that the truncation of a standard thin disk may not be required in order to drive relativistic jets. Truncation may aid in creating stable poloidal magnetic fields, for instance (Reynolds et al. 2006), and for such a reason may amplify jet power, but the results of this program suggest that truncation is not a requirement. Taken at face value, our results suggest that a standard thin accretion disk that extends to the ISCO can drive relativistic jets. This action may be mediated by the hard X-ray corona, or course, and the onset of jet production as black holes transit from high/soft or intermediate states into the low/hard state may have more to do with changes in the corona than changes in the disk.

The second central result of this program is that the accretion disk is connected to the relativistic jet: in both phenomenological and physical spectral fits, the disk flux and disk temperature are found to be correlated with radio flux. These parameters are likely tied to the mass accretion rate through the disk and may serve as evidence of a connection between the accretion rate in the disk and the radio luminosity of the jet. Jet radio luminosity is an imperfect proxy for jet power (Allen et al. 2006), but this result may also nominally indicate a link between the mass accretion rate in the disk and jet power.

It is also important to note and explore connections between the jet and the hard X-ray corona. Radio flux is found to correlate positively with parameters such as the photon index of the power-law spectral component. Our results also suggest that the corona may have an indirect role, at least, in driving relativistic jets. Like other studies that require a disk close to the ISCO in the low/hard state, our results suggest that the hard X-ray corona is not a central, optically thin, Comptonizing region within a truncated disk. This does not rule out Comptonization; indeed, the high-energy turnover seen in these spectra is a hallmark of Comptonization (see, e.g., Makishima et al. 2008; Torii et al. 2011). Rather, a different geometry is merely required to describe these observations.

Beloborodov (1999) developed a model for accretion and ejection in Cygnus X-1, wherein hard X-rays are generated in mildly relativistic plasma moving away from the disk. Especially in light of later work by Markoff et al. (2001, 2005), it is natural to associate the outflowing relativistic plasma with the base of the radio jet. In the Beloborodov (1999) model, the ejected material is initially lifted above the disk by magnetic flaring activity generated by the magneto-rotational instability (MRI), and may be ejected by the pressure of reflected radiation. Thus, this model predicts a connection involving the disk, corona, disk reflection, and the jet.

As noted previously, positive correlations are found between disk flux and temperature, and radio flux. These disk parameters are likely tied to the mass accretion rate, which is of course 
linked to the MRI. Thus, these simple correlations are consistent with one aspect of the plasma ejection model of Beloborodov (1999). However, a different correlation may offer even deeper insights and more specific support: the data show a positive correlation between the reflected X-ray flux and the radio flux (see Figure 18). A weaker correlation between disk ionization and radio flux is also found (see Table 7 and Figure 16). The positive correlation between reflected flux and radio flux is consistent with the prediction that plasma blobs are ejected partly due to the pressure of the reflected radiation. Note that reflected flux strongly dominates over direct disk flux in all of our spectral fits (see Table 4). Thus, our results may offer specific, detailed support for the plasma ejection framework described in Beloborodov (1999).

A further prediction of the plasma ejection model is that the $\mathrm{X}$-ray power-law spectral index, $\Gamma$, is related to the velocity of the outflowing plasma via $\Gamma=1.9 / \sqrt{B}$, where $B=\gamma(1+\beta)$ (here, $\beta=v / c$ and $\gamma=1 / \sqrt{1-\beta^{2}}$ ). Fits to the Suzaku data with blurred reflection models are consistent with a power-law index of $\Gamma=1.4$, corresponding to $v / c \simeq 0.3$. It is worth noting that this velocity is the escape speed for a distance of $z \simeq 20 \mathrm{GM} / \mathrm{c}^{2}$ from the black hole. It is also notable that this velocity is broadly consistent with the plasma velocity $(v / c=0.4)$ assumed at the base of the jet in Markoff et al. (2005). In that work, emission from the base of a jet is shown to naturally include Comptonization and shown to generate disk reflection that is consistent with observations. An important aspect of these broadband jet models is that injected particles are accelerated into a power-law tail at a height of $z=$ 10-100 $G M / c^{2}$ above the disk (see below).

Although fits with the phenomenological spectral models appear to confirm prior evidence of feedback between the disk and corona in the form of the $R-\Gamma$ correlation (Zdziarski et al. 2003; see Figures 10 and 11, and Tables 5 and 6), more physical and self-consistent fits with the blurred disk reflection spectrum find an anti-correlation between the incident and reflected flux (see Section 4.3, Tables 6 and 7, and Figure 19). This can be explained in terms of gravitational light bending close to a spinning black hole. Depending on the vertical displacement of hard X-ray emission relative to a black hole, more or less hard flux may be beamed onto the disk, leading to a nonlinear relationship between direct and reflected flux (Miniutti \& Fabian 2004). This sort of nonlinear relationship has been observed in some Seyfert-1 AGNs, including MCG-6-30-15 (Miniutti et al. 2007), but also in the stellar-mass black hole XTE J1650-500 (Miniutti et al. 2004; Rossi et al. 2005).

Relativistic blurring and light bending may also explain one point of disparity between the results of this program and Beloborodov (1999). The outflowing corona model was partly motivated by results suggesting low reflection fractions in stellar-mass black holes in the low/hard state. The model was developed before extremely blurred lines were observed in stellar-mass black holes using CCD spectrometers, however, and before potential evidence of light bending had been observed in flux trends. After blurring, a reflection model is not as sharp, and a higher reflection flux may be required to fit peaks in the data. Moreover, if light bending is even somewhat important, it is difficult to ascertain how much flux is seen directly versus how much is focused onto the disk. Although most of our fits are not consistent with a low reflection fraction, the basics of outflowing corona models do not depend on the reflection fraction.

The results of fits to the disk continuum, disk line, and reflection spectrum are given in Section 4. Factoring in all of the models and results, and emphasizing fits where strong constraints are obtained, the data suggest that Cygnus X-1 has a spin in the range of $0.6 \leqslant a \leqslant 0.99$. This rather large range derives primarily from lower spin values found based on fits to the disk continuum. However, the models used were less physical than the relativistic reflection models that were employed. The reflection fits strongly prefer a near-maximal spin for Cygnus $\mathrm{X}-1$. This is at odds with the results of recent reflection fits to some XMM-Newton spectra of Cygnus X-1 (Miller et al. 2009b); however, those spectra may give falsely low spin values owing to photon pile-up effects (see, e.g., Miller et al. 2010). However, the high spin implied in our fits is consistent with more recent and more detailed work by Gou et al. (2011), which places a $3 \sigma$ lower limit of $a>0.91$. It is also consistent with recent work by Duro et al. (2011), which measures $a=0.88_{-0.11}^{+0.07}$ using a new mode of the XMM-Newton/EPIC-pn camera, and a measurement of $a=0.97_{-0.02}^{+0.01}$ based on fits to Suzaku spectra (Fabian et al. 2012). Thus, anisotropic hard X-ray emission due to gravitational light bending, if not also bulk flows (see above), is plausible in Cygnus $\mathrm{X}-1$.

In this work, an emissivity of $r^{-3}$ was held fixed in all fits with "Laor" and "kdblur." This action was taken partly because steeper indices were not required by the data when considering a simple power-law functional form, and partly because spin estimates made by Duro et al. (2011) also suggest this emissivity is reasonable. However, an index of $q=3$ is at odds with the steeper indices sometimes obtained in black holes where fits suggest high spin values, such as MCG-6-30-15 (Brenneman \& Reynolds 2006; Miniutti et al. 2007), 1H 0707-495 (Fabian et al. 2009; Wilkins \& Fabian 2011), XTE J1650-500 (Miller et al. 2009b), GX 339-4 (Miller et al. 2008; Reis et al. 2008), and XTE J1550-564 (e.g., Miller et al. 2009b; Steiner et al. 2011). It is interesting to note that emissivity may be positively correlated with Eddington fraction, since most of the high $q$ values are recorded in "intermediate" and "very high" states (for a review of the stellar-mass black hole cases, see Miller et al. 2009b).

Motivated by the disk reflection spectrum seen in $1 \mathrm{H}$ 0707-479 (Fabian et al. 2009), Wilkins \& Fabian (2011) have calculated how the emissivity index should vary with black hole spin for different idealizations of the hard X-ray corona. It is found that the emissivity can be represented as a twice-broken power law, with a break radius at or within $6 G M / c^{2}$. Especially if the black hole spins rapidly and the hard X-ray source is nearby, the inner emissivity index is expected to be steep $(q>3)$, while the outer index is expected to be flatter. However, if the source of hard X-ray emission is $z \simeq 10-30 \mathrm{GM} / \mathrm{c}^{2}$ above a spinning black hole, $q=3$ is expected. This model implies that the source of hard X-ray emission in the low/hard state of Cygnus X-1 may be approximately $10-30 G M / c^{2}$ above the disk. Preliminary fits with the broken power-law emissivity index suggest that Cygnus X-1 may follow the predictions of Wilkins \& Fabian (2011); a fit to a single spectrum of Cygnus $\mathrm{X}-1$ assuming an on-axis point source is detailed in Fabian et al. (2012). Our assumption of a less extreme emissivity is consistent with a corona that has a small but finite spatial extent.

At a height of $\simeq 20 \mathrm{GM} / \mathrm{c}^{2}$, the light bending model independently predicts that reflected flux should be anti-correlated with the incident power-law flux (see the top panel of Figure 2 in Miniutti \& Fabian 2004). Figure 19 plots the reflected flux measured in our spectral fits versus the measured power-law flux. The anti-correlation between these parameters recorded in Table 8 is clear in the plot as well. At heights greater 
than $\simeq 20 \mathrm{GM} / \mathrm{c}^{2}$, light bending is predicted to be inefficient (Miniutt \& Fabian 2004). Thus, if light bending plays a role in driving the variability and reflection seen in Cygnus $\mathrm{X}-1$, a height of $z \simeq 20 \mathrm{GM} / \mathrm{c}^{2}$ is required; both larger and smaller values are inconsistent with the observed flux pattern. It is not clear how else the anti-correlation between reflected and incident flux could be explained; changes in the disk ionization are insufficient and would produce a positive correlation as the disk albedo increases with ionization.

Thus, taken as a whole, the results of this program suggest a simple and self-consistent picture that can be summarized in stages. (1) Material in the corona (jet base) may be initially lifted from the disk by MRI. This is consistent with observed correlations between radio flux and disk flux and temperature. (2) The material can then be accelerated at least partially through the pressure of reflected radiation. This is supported by a correlation between radio flux and reflected X-ray flux. (3) Comptonization in the plasma above the disk produces the hard X-ray spectrum in a manner that links the bulk velocity of the outflow and the X-ray power-law index. The observed power-law indices are suggestive of a moderately relativistic plasma, with $v / c \simeq 0.3$. (4) Last, the relationship between direct and reflected hard X-ray flux, and the disk emissivity index are driven by the distance between the hard X-ray source and black hole. The reflection fitting results bear the hallmarks of light bending and suggest hard X-ray emission from $z \simeq 20 \mathrm{GM} / \mathrm{c}^{2}$ above the black hole. This distance coincides with the zone where hard X-rays are generated in jet-based models for black hole SEDs (Markoff et al. 2005) and is consistent with the lag timescales uncovered in advanced X-ray variability studies of Cygnus X-1 (Uttley et al. 2011; also see Wilkinson \& Uttley 2009).

The results discussed here imply that hard X-ray emission is produced in a fairly compact region. It is difficult to make an independent test of the size of the emission region in stellarmass black holes; however, microlensing makes such tests possible in AGNs. Recent microlensing results in quasars find that the hard X-ray emission regions have half-light radii of only $10 G M / c^{2}$ (Chartas et al. 2009; Dai et al. 2010; Chen et al. 2011). These results provide some important observational support for compact, central, hard X-ray emission regions.

Our results offer some insights into the nature of the disk, the hard X-ray corona, and jet production in stellar-mass black holes in the low/hard state. Moreover, they serve to highlight some natural connections between the plasma ejection model of Beloborodov (1999), broadband jet models (e.g., Markoff et al. 2005), and the effects of gravitational light bending on hard $\mathrm{X}$-rays produced close to a spinning black hole (e.g., Miniutti $\&$ Fabian 2004). Can this confluence of models and processes also explain the absence of jets in other black hole states?

As noted above, high values of the disk emissivity index are preferentially found in the "intermediate" and "very high" spectral states. In these phases, disk reflection is especially strong. Both of these properties are consistent with hard X-ray emission originating closer to the black hole, perhaps at $z \simeq$ $5-10 G M / c^{2}$, where the escape velocity is higher, making it harder to eject a plasma. Moreover, the Beloborodov (1999) coronal ejection model predicts a lower plasma velocity for the steeper spectral slopes that are typically observed in "intermediate" and "very high" spectral states. Reflection is weakest, and jets are weakest (or quenched entirely) in disk-dominated "high/soft" states (see, e.g., Russell et al. 2011), where powerlaw fits to hard X-ray spectra generally yield the steepest slopes. This is again consistent with clues to the nature of disk-corona-jet coupling that emerge from Cygnus X-1.

Livio et al. (2003) describe a model for disk-jet coupling in stellar-mass black holes and AGNs that relies on fluctuations in the poloidal magnetic field emerging from the accretion disk. This model builds upon the work of Blandford \& Payne (1982) but makes specific predictions and comparisons to individual sources such as GRS $1915+105$. Our results are broadly compatible with this model, especially if it is a combination of the disk flux $(\dot{m})$, temperature, and ionization that serves to modulate poloidal magnetic fields. This model does not address the role of disk reflection in setting disk ionization, nor in helping to accelerate gas that has been lifted off of the disk. Thus, it is not necessarily in conflict with the models described by Beloborodov (1999), Markoff et al. (2005), and Miniutti \& Fabian (2004). It is possible that radiation pressure from reflected radiation and magnetocentrifugal forces may work in tandem to drive jet plasma away from the disk.

In many respects, Seyfert-1 AGNs resemble stellar-mass black holes in the low/hard or "very high" state: their power-law indices are broadly similar, disk reflection spectra are prominent in many cases, and power spectra show band-limited noise with characteristic frequencies. In terms of their Eddington fraction, however, most Seyfert-1 AGNs would be better associated with the "low/hard" state (see, e.g., Blustin et al. 2005). It is interesting to briefly consider how the results of this study of Cygnus X-1 might impact our understanding of Seyfert AGNs.

Unlike stellar-mass black holes, where the flux in hard $\mathrm{X}$-rays or reflected X-rays can dominate the total luminosity, UV emission from the much cooler disk around a supermassive black hole dominates the radiative output of Seyferts. Radiation pressure from the disk itself should strongly dominate over pressure from reflected emission. At present, no comparable study of a Seyfert has been performed that could confirm or reject the correlation between reflected $\mathrm{X}$-ray flux and radio flux found in Cygnus X-1. However, coordinated studies of AGNs from different classes are now being undertaken. Recent efforts to obtain contemporaneous X-ray and radio monitoring observations of the Seyfert-1 NGC 4051, for instance, reveal a mode of disk-jet coupling that may differ from stellar-mass black holes in the low/hard state (King et al. 2011; Jones et al. 2011). Note, however, that jet-based spectral models do provide a good description of the broadband SED of NGC 4051 (Maitra et al. 2011). Low-luminosity AGNs (LLAGNs) may be a better comparison to Cygnus X-1 in the low/hard state, although it is more difficult to study the inner accretion disk in such sources. Nevertheless, radio and X-ray flux monitoring of the LLAGN NGC 7213, for instance, has shown a coupling similar to that revealed in stellar-mass black holes in the low/hard state (Bell et al. 2011).

Last, it is worth remarking on the limited scope of this analysis and offering some brief comparisons to other recent efforts to model black hole spectra. Nowak et al. (2011) have shown that a variety of simple phenomenological models, complex Comptonization models, and disk reflection models can all provide good fits to spectra of Cygnus X-1. Both disk reflection and Comptonization must be at work, at some level, but models differ as to the degree and the specifics of the accretion flow geometry. This analysis has focused on reflection; it is not perfectly consistent in its treatment of Comptonization and the roll-over at high energy. Indeed, fully consistent descriptions of Comptonization prove to be difficult. Some recent efforts to model spectra from Cygnus X-1 have taken the extraordinary 
step of using two Comptonization components: the electron temperatures in two "compps" components are linked, but each region has a distinct optical depth (e.g., Makishima et al. 2008). If the gravitational potential sets the local electron temperature, then these regions must be close to each other. Their different optical depths imply different densities and internal pressures, however, and they would diffuse into each other on a short interval driven by the local orbital timescale. The regions could potentially be kept distinct via some form of magnetic confinement, but that evokes the geometry that is implied by our fits-one of a corona with a strong magnetic component. Though different families of models still differ considerably, it is possible that they are on a convergent path.

\section{CONCLUSIONS}

1. Variations in the radial extent of the inner accretion disk in the low/hard state of Cygnus X-1 do not appear to drive changes in the relativistic jet.

2. However, the flux and temperature of the inner accretion disk-parameters likely tied to $\dot{m}$-positively correlate with radio flux, suggesting a true disk-jet coupling.

3. The stability of the inner disk in the low/hard state in Cygnus X-1 suggests that it sits close to the ISCO. This is supported by the detailed results of direct spectral fits.

4. The implication of a disk close to the ISCO in the low/hard state would signal that standard thin disks can power relativistic jets.

5. Factoring in both phenomenological and more physical fits to the disk continuum and reflection spectrum, and making cuts on measurement quality, the inner disk radii suggest a spin of $0.6 \leqslant a \leqslant 0.99$, consistent with some other recent results (Gou et al. 2011; Duro et al. 2011; also see Miller et al. 2005). Blurred, ionized reflection fits prefer a near-maximal spin.

6. Some fundamental predictions of a simple disk reflection geometry appear to be confirmed in phenomenological X-ray spectral fits. More physical and more self-consistent fits with a blurred reflection spectrum may require an anisotropic hard X-ray source, among other complexities.

7. A positive correlation between the reflected X-ray flux and the radio flux suggests a connection between disk reflection and jet power. This is consistent with a plasma ejection model for the corona (or, jet base) developed by Beloborodov (1999).

8. An anti-correlation between the reflected and direct powerlaw flux is consistent with a model in which gravitational light bending close to a black hole partially drives the flux variations (e.g., Miniutti \& Fabian 2004). The anticorrelation between these fluxes suggests a source height of $z \simeq 20 G M / c^{2}$, which is consistent with an independent prediction based on the disk emissivity parameter.

9. Hard X-ray production at a height of $z \simeq 20 \mathrm{GM} / \mathrm{c}^{2}$ is consistent with recent time lags found in X-ray variability studies (Uttley et al. 2011) and consistent with theoretical predictions for the region of initial particle acceleration in the base of a jet (Markoff et al. 2005).

We thank the anonymous referee for comments that have improved this manuscript. We thank Koji Mukai, Robert Petre, Kazuhisa Mitsuda, and the entire Suzaku team for their help in executing these observations. We thank Elena Gallo,
Dipankar Maitra, Chris Reynolds, Mark Reynolds, and Mateusz Ruszkowski for helpful discussions.

\section{REFERENCES}

Anders, E., \& Grevesse, N. 1989, Geochim. Cosmochim. Acta, 53, 197

Allen, S. W., Dunn, R. J. H., Fabian, A. C., Taylor, G. B., \& Reynolds, C. S. 2006, MNRAS, 372, 21

Balucinska-Church, M., Church, M. J., Charles, P. A., et al. 2000, MNRAS, 311,861

Bardeen, J. M., \& Petterson, J. A. 1975, ApJ, 195, L65

Beckwith, K., \& Done, C. 2004, MNRAS, 352, 353

Bell, M. E., Tzioumis, T., Uttley, P., et al. 2011, MNRAS, 411, 402

Beloborodov, A. 1999, ApJ, 510, L123

Blandford, R. D., \& Payne, D. G. 1982, MNRAS, 199, 883

Blandford, R. D., \& Znajek, R. L. 1977, MNRAS, 179, 433

Blum, J. L., Miller, J. M., Fabian, A. C., et al. 2009, ApJ, 706, 60

Blustin, A. J., Page, M. J., Fuerst, S. V., Branduardi-Raymont, G., \& Ashton, C. E. 2005, A\&A, 431, 111

Brenneman, L., \& Reynolds, C. S. 2006, ApJ, 652, 1028

Brocksopp, C., Tarasov, A. E., Lyuty, V. M., \& Roche, P. 1999, A\&A, 343, 861

Calvet, N., Hartmann, L., \& Kenyon, S. 1993, ApJ, 402, 623

Chartas, G., Kochanek, C. S., Dai, X., Poindexter, S., \& Garmire, G. 2009, ApJ, 693, 174

Chen, B., Dai, X., Kochanek, C. S., et al. 2011, ApJ, 740, L34

Dai, X., Kochanek, C. S., Chartas, G., et al. 2010, ApJ, 709, 278

Dovciak, M., Karas, V., \& Yaqoob, T. 2004, ApJS, 153, 205

Duro, R., Dauser, T., Wilms, J., et al. 2011, A\&A, 533, L3

Ebisawa, K., Ueda, Y., Inoue, H., Tanaka, Y., \& White, N. E. 1996, ApJ, 467, 419

Esin, A. A., McClintock, J. E., \& Narayan, R. 1997, ApJ, 489, 865

Fabian, A. C., Wilkins, D. R., Miller, J. M., et al. 2012, MNRAS, 424, 217

Fabian, A. C., Zoghbi, A., Ross, R. R., et al. 2009, Nature, 459, 540

Falcke, H., Kording, E., \& Markoff, S. 2004, A\&A, 414, 895

Fender, R., Gallo, E., \& Russell, D. 2010, MNRAS, 406, 1425

Frontera, F., Palazzi, E., Zdziarski, A. A., et al. 2001, ApJ, 546, 1027

Gallo, E., Fender, R., \& Pooley, G. 2003, MNRAS, 344, 60

George, I. M., \& Fabian, A. C. 1991, MNRAS, 249, 352

Gierlinski, M., Done, C., \& Page, K. 2009, MNRAS, 392, 1106

Gies, D. R., Bolton, C. T., Blake, R. M., et al. 2008, ApJ, 678, 1237

Gilfanov, M., Churazov, E., \& Revnivtsev, M. 1999, A\&A, 352, 182

Gou, L., McClintock, J. E., Reid, M. J., et al. 2011, ApJ, 742, 85

Gultekin, K., Cackett, E. M., Miller, J. M., et al. 2009, ApJ, 706, 404

Hanke, M., Wilms, J., Nowak, M. A., et al. 2009, ApJ, 630, 330

Jones, S., McHardy, I., Moss, D., et al. 2011, MNRAS, 412, 2641

Junor, W., Biretta, J. A., \& Livio, M. 1999, Nature, 401, 891

King, A. L., Miller, J. M., Cackett, E. M., et al. 2011, ApJ, 729, 19

Kraemer, S. B., Crenshaw, D. M., Gabel, J. R., et al. 2006, ApJS, 167, 161

Kubota, A., Dotani, T., Cottam, J., et al. 2007, PASJ, 59, 185

Laor, A. A. 1991, ApJ, 376, 90

Livio, M., Pringle, J., \& King, A. R. 2003, ApJ, 593, 184

Maccarone, T. 2002, MNRAS, 336, 1371

Magdziarz, P., \& Zdziarski, A. A. 1995, MNRAS, 273, 837

Maitra, D., Miller, J. M., Markoff, S., \& King, A. L. 2011, ApJ, 735, 107

Makishima, K., Takahashi, H., Yamada, S., et al. 2008, PASJ, 60, 585

Markoff, S., Falcke, H., \& Fender, R. 2001, A\&A, 372, L25

Markoff, S., Nowak, M., \& Wilms, J. 2005, ApJ, 635, 1203

Marscher, A. P., Jorstad, S. G., D’Arcangelo, F. D., et al. 2008, Nature, 452, 966

Mauche, C., \& Raymond, J. C. 2000, ApJ, 541, 924

McClintock, J. E., Narayan, R., Gou, L., et al. 2010, in AIP Conf. Ser. 1248, Xray Astronomy 2009: Present Status, Multi-wavelength Approach and Future Perspectives, ed. W. Hendrik, G. Lewin, \& M. van der Klis (Melville, NY: AIP), 101

McNamara, B. R., Kazemzadeh, F., Rafferty, D. A., et al. 2009, ApJ, 689, 594 Merloni, A., Heinz, S., \& Di Matteo, T. 2003, MNRAS, 345, 1057

Merloni, A., Ross, R., \& Fabian, A. C. 2000, MNRAS, 313, 193

Miller, J. M. 2007, ARA\&A, 45, 441

Miller, J. M., Cackett, E. M., \& Reis, R. C. 2009a, ApJ, 707, L77

Miller, J. M., D’Aì, A., Bautz, M. W., et al. 2010, ApJ, 724, 1441

Miller, J. M., Fabian, A. C., Wijnands, R., et al. 2002, ApJ, 578, 348

Miller, J. M., Homan, J., Steeghs, D., et al. 2006a, ApJ, 653, 525

Miller, J. M., Miller, M. C., \& Reyonlds, C. S. 2011, ApJ, 731, L5

Miller, J. M., Raymond, J., Fabian, A. C., et al. 2006b, Nature, 441, 953

Miller, J. M., Raymond, J., Reynolds, C. S., et al. 2008, ApJ, 680, 1359

Miller, J. M., Reynolds, C. S., Fabian, A. C., Miniutti, G., \& Gallo, L. C. 2009b, ApJ, 697, 900 
Miller, J. M., Wojdowski, P., Schulz, N. S., et al. 2005, ApJ, 620, 398 Mineshige, S., Hirano, A., Kitamoto, S., Yamada, T., \& Fukue, J. 1994, ApJ, 426, 308

Miniutti, G., \& Fabian, A. C. 2004, MNRAS, 349, 1435

Miniutti, G., Fabian, A. C., Anabuki, N., et al. 2007, PASJ, 59, 315

Miniutti, G., Fabian, A. C., \& Miller, J. M. 2004, MNRAS, 351, 466

Mitsuda, K., Inoue, H., Koyama, K., et al. 1984, PASJ, 36, 741

Nandra, K., O’Neill, P. M., George, I. M., \& Reeves, J. N. 2007, MNRAS, 382 , 194

Narayan, R., \& McClintock, J. E. 2012, MNRAS, 419, L69

Nayakshin, S., \& Kallman, T. R. 2001, ApJ, 546, 406

Nowak, M. A. 2009, Scripts Available Online Via space.mit.edu/CXC/software/ suzaku/index.html

Nowak, M. A., Hanke, M., Trowbridge, S. N., et al. 2011, ApJ, 728, 13

Orosz, J. A., McClintock, J. E., Aufdenberg, J. P., et al. 2011, ApJ, 742, 84

Pooley, G. G., \& Fender, R. 1997, MNRAS, 292, 925

Pooley, G. G., Fender, R., \& Brocksopp, C. 1999, MNRAS, 302, L1

Reid, M. J., McClintock, J. E., Narayan, R., et al. 2011, ApJ, 742, 83

Reis, R. C., Fabian, A. C., \& Miller, J. M. 2010, MNRAS, 402, 836

Reis, R. C., Fabian, A. C., Ross, R. R., et al. 2008, MNRAS, 387, 1489

Reis, R. C., Miller, J. M., Reynolds, M. T., Fabian, A. C., \& Walton, D. J. 2012, ApJ, 751, 34

Reynolds, C. S., Garofalo, D., \& Begelman, M. C. 2006, ApJ, 651, 1023

Reynolds, M. T., \& Miller, J. M. 2012, ApJ, submitted (arXiv:1112.2249)

Reynolds, M. T., Miller, J. M., Homan, J., \& Miniutti, G. 2010, ApJ, 709, 358

Ross, R. R., \& Fabian, A. C. 2005, MNRAS, 358, 211

Rossi, S., Homan, J., Miller, J. M., \& Belloni, T. 2005, MNRAS, 360, 763
Russell, D. M., Miller-Jones, J. C. A., Maccarone, T. J., et al. 2011, ApJ, 739, L19

Rykoff, E. S., Miller, J. M., Steeghs, D., \& Torres, M. A. P. 2007, ApJ, 666, 1129

Schulz, N. S., Cui, W., Canizares, C. R., et al. 2002, ApJ, 565, 1141

Shimura, T., \& Takahara, F. 1995, ApJ, 445, 780

Sikora, M., Stawarz, L., \& Lasota, J.-P. 2007, ApJ, 658, 815

Sobczak, G. J., McClintock, J. E., Remillard, R. A., et al. 2000, ApJ, 544, 993

Sowers, J. W., Gies, D. R., Bagnuolo, W. G., et al. 1998, ApJ, 506, 424

Steiner, J. F., McClintock, J. E., Remillard, R. A., et al. 2010, ApJ, 718, L117

Steiner, J. F., Reis, R. C., McClintock, J. E., et al. 2011, MNRAS, 416, 941

Stirling, A. M., Spencer, R. E., de la Force, C. J., et al. 2001, MNRAS, 327, 1273

Tomsick, J. A., Yamaoka, K., Corbel, S., et al. 2009, ApJ, 707, L87

Torii, S., Yamada, S., Makishima, K., et al. 2011, PASJ, 63, 771

Uttley, P., Wilkinson, T., Cassatella, P., et al. 2011, MNRAS, 404, L60

Wilkins, D. R., \& Fabian, A. C. 2011, MNRAS, 414, 1269

Wilkinson, T., \& Uttley, P. 2009, MNRAS, 397, 666

Wilms, J., Allen, A., \& McCray, R. 2000, ApJ, 542, 914

Wilms, J., Nowak, M. A., Pottschmidt, K., Pooley, G. G., \& Fritz, S. 2006, A\&A, 447,245

Zdziarski, A. A., Lubinski, P., Gilfanov, M., \& Revnivtsev, M. 2003, MNRAS, 342,355

Zdziarski, A. A., Skinner, G. K., Pooley, G. G., \& Lubinski, P. 2011, MNRAS, 416, 1324

Zhang, S. N., Cui, W., \& Chen, W. 1997, ApJ, 482, L155

Zwart, J. T. L., Barker, R. W., Biddulph, P., et al. 2008, MNRAS, 391, 1545 